\title{
Multiseparability and Superintegrability for Classical and Quantum Systems
}

\author{
W. Miller, Jr. \\ Institute for Mathematics and its Applications \\ University of Minnesota, \\ Minneapolis, Minnesota, 55455, U.S.A.
}

February 17, 2000

\begin{abstract}
It has long been known that there are potentials on $n$-dimensional constant curvature spaces for which a given Hamiltonian system in classical mechanics, and Schrödinger equation in quantum mechanics, admits solutions via separation of variables in more than one coordinate system. Smorodinsky, Winternitz et.al., initiated the methodical search for such potentials in two and three dimensions, and there has been a considerable amount of work for various examples. Such a system is called maximal in dimension $n$ if there exist $2 n-1$ functionally independent integrals of motion. In some papers, these systems are called superintegrable.

In the first part of this paper we outline the basic ideas relating to the notion of superintegrable potentials. The energy observable is degenerate for potentials of this type and the corresponding intergrals of motion that arise from the simultaneous separability close quadratically under repeated commutation. We give examples of these systems and indicate how superintegrability can be of use, particularly in relation to bound states. Virtually all of the special functions of mathematical physics (in one and several variables) arise in this study and formulas expanding one type of special function as a series in another type emerge as a byproduct.

Finally, we describe how one can, in principle, classify all such systems and deduce the structure of the quadratic algebra. Many of the results reported
\end{abstract}


here were obtained in collaboration with E.G. Kalnins and G.S. Pogosyan.

\section{Introduction}

It has long been known that Schrödinger's equation with certain special potentials can admit (multiplicative) separation of variables in more than one coordinate system. This is intimately related to the notion of superintegrability, $[1,2,3]$. This subject has been studied by a number of authors, based on the use of the corresponding differential equations that that are implied by the requirement of simultaneous separability, $[4,5,6,7,8,9,10,11,12,13,14$, 15]. Specifically, superintegrability means that for a Schrödinger equation in dimension $n$ there exist $2 n-1$ functionally independent quantum mechanical second-order observables (i.e., second-order self-adjoint operators that commute with the Hamiltonian). There is an analogous concept of superintegrability for classical mechanical systems. This relates to the corresponding additive separation of variables of the Hamilton-Jacobi equation. Furthermore, one observes that if we do have simultaneous separability then the resulting constants of the motion close quadratically under repeated application of the Poisson bracket, [11]. We also know that for spaces of constant curvature separable coordinate systems of the free motion are described by quadratic elements of the corresponding first order symmetries, $[16,17,18]$.

Although concrete examples of superintegrable systems are easily at hand, a complete classification of all such systems has presented major difficulties. How can one be sure that all systems for free motion have been found? (For example, Rañada's classification [15] omits our system 5 below.) Once these are determined, how can one be sure that the most general additive potential term has been calculated?

Here we will present the background information to understand the problem, and its importance, and present a new approach to its solution, with details for two dimensional complex Euclidean space.

Consider an $n$-dimensional Riemannian manifold $R_{n}$. (In most of the following we will assume that $R_{n}$ is a space of constant curvature, for that is the case where the most interesting and rich applications arise.) In local

coordinates $q_{1}, \cdots, q_{n}$ the contravariant metric tensor is $\left(g^{j k}(\mathbf{q})\right)$. Let $V(\mathbf{q})$ be a potential function on $R_{n}$. The corresponding Hamilton-Jacobi equation 
is

$$
\mathcal{H}(\mathbf{q}, \mathbf{p})=E
$$

where

$$
\mathcal{H}(\mathbf{q}, \mathbf{p})=\sum_{j, k=1}^{n} g^{j k}(\mathbf{q}) p_{j} p_{k}+V(\mathbf{q})=\sum_{j, k=1}^{n} g^{j k}(\mathbf{q}) \frac{\partial S}{\partial q_{j}} \frac{\partial S}{\partial q_{k}}+V(\mathbf{q})
$$

and $S(\mathbf{q})$ is the action function, [19].

The quantum analog of this classical system is given by the Schrödinger equation

$$
H \Psi(\mathbf{q})=E \Psi(\mathbf{q})
$$

where in local coordinates

$$
H=\Delta_{n}+V(\mathbf{q}), \quad \Delta_{n}=\frac{1}{\sqrt{g}} \sum_{j, k=1}^{n} \frac{\partial}{\partial q_{j}} \cdot\left(\sqrt{g} g^{j k}\right) \frac{\partial}{\partial q_{k}}
$$

and $g=\operatorname{det}\left(g^{j k}\right)^{-1}$.

Recall that a complete integral $S\left(\mathbf{q}, \lambda_{1}, \cdots, \lambda_{n}\right)$ of the Hamilton-Jacobi equation solves the associated classical mechanical system $[19,20]$. (A complete integral is a solution of (1) such that locally

$$
\operatorname{det}\left(\frac{\partial^{2} S}{\partial q_{j} \partial \lambda_{k}}\right) \neq 0
$$

Any solution of the Hamilton-Jacobi equation via (additive) separation of variables

$$
S\left(\mathbf{q}, \lambda_{1}, \cdots, \lambda_{n}\right)=\sum_{j=1}^{n} S^{(j)}\left(q_{j}, \lambda\right)
$$

where $\lambda_{1}=E, \lambda_{2}, \cdots, \lambda_{n}$ are the separation constants, yields a complete integral.

Similarly, in the quantum case, if the Schrödinger equation $H \Psi=E \Psi$ (multiplicatively) separates in the coordinates $\mathbf{q}$ then we can write

$$
\Psi(\mathbf{q})=\Pi_{j=1}^{n} \Psi^{(j)}\left(q_{j}, \lambda\right)
$$

and this ansatz allows the decomposition of (3) into $n$ ordinary differential equations, one for each of the factors $\Psi^{(j)}$. Many of the special functions of 
mathematical physics occur as solutions of these ordinary differential equations.

For orthogonal coordnates $\mathbf{q}$ on an $n$-dimensional constant curvature space (i.e., such that $g^{j k}=0$ for $j \neq k$ ) one can show that the HamiltonJacobi equation is additively separable if and only if the Shrödinger equation is multiplicatively separable. (See [21] for a discussion of the relationship for general Riemannian manifolds.) We shall see that superintegrability is closely linked to symmetry properties of (1) and (3), and to separation of variables (special function) solutions of (3).

At this point it is useful to summarize briefly the history of the symmetry/special function approach to solving the Schrödinger equation (3). (Superintegrability is just one of the latest chapters.) Beginning with the introduction of the Schrödinger equation in the 1930's, and continuing until the 1960's, the main emphasis was on the study of Lie symmetry groups of unitary operators that commuted with the Hamiltonian $H$, hence mapped solutions of (3) to solutions. At the Lie algebra level, one looked for algebras of first-order differential operators $L$ that commuted with $H$ : $[L, H] \equiv$ $L H-H L=0$. (Again $L$ maps solutions $\Psi$ of (3) to solutions.) This led to studies of rotationally invariant potentials and the theory of spherical harmonics [22].

A related concept was that of dynamical symmetry groups or Lie algebras. The idea was to imbed $H$ as an element of a Lie algebra of first and second-order differential operators. The representation theory of the Lie algebra could then be used to derive information about the eigenvalues and eigenvectors of $H$. The harmonic oscillator and the Morse potential were treated in this way. The so-called factorization method for solving the Schrödinger equation is related to this approach [23]. Among the special functions that arise and whose properties can be studied from this connection are Bessel functions and (more generally) hypergeometric functions. (More recently, $q$-analogs of the dynamical symmetry algebra approach have led to $q$-hypergeometric functions [24].)

Since the 1960's we have been in the "Cheshire Cat" era, [25]. In the most recent theories relating integrabilty, superintegrability and variable separation, the Lie groups and algebras have disappeared, but their grin persists, $\smile$. The focus here is on second order constants of the motion (symmetry operators that are built out of products of first order Lie symmetries for the zero potential problem) and their connection with variable separation for 
both the Hamilton-Jacobi and Schrödinger equations. One of the seminal papers in this regard was by Smorodinsky et al. [5]. Some other contributions are, for example, by Shapovalov, Kalnins and Miller, and Winternitz $[26,27,28]$.These approaches also exploit the maximum symmetry of the physical system, but no longer in terms of Lie algebras of operators.

To examine these ideas, let us start with a classical system

$$
\mathcal{H}=\sum g^{j k} p_{j} p_{k}+V(\mathbf{q})
$$

where the $p_{j}$ are the momenta conjugate to the coordintes $q_{j}$. Recall that the Poisson bracket of two functions $f_{h}(\mathbf{q}, \mathbf{p}), h=1,2$ is the function

$$
\left\{f_{1}, f_{2}\right\}(\mathbf{q}, \mathbf{p})=\sum_{j=1}^{n}\left(\frac{\partial f_{1}}{\partial q_{j}} \frac{\partial f_{2}}{\partial p_{j}}-\frac{\partial f_{1}}{\partial p_{j}} \frac{\partial f_{2}}{\partial q_{j}}\right)
$$

[20]. A second-order constant of the motion for (6) is a function

$$
\mathcal{L}=\sum a^{j k}(\mathbf{q}) p_{j} p_{k}+W(\mathbf{q}), \quad a^{j k}=a^{k j},
$$

such that $\{\mathcal{L}, \mathcal{H}\}=0$.

Note that the null space of the map

$$
T: \quad d f(\mathbf{q}, \mathbf{p}) \rightarrow\{f, \mathcal{H}\}(\mathbf{q}, \mathbf{p})
$$

is $2 n-1$ dimensional. Thus (locally) there are $2 n-1$ functionally independent constants of the motion (but not necessarily second-order). For the purposes of this paper we adopt the following definitions. We say that the classical system $\mathcal{H}=E$ is superintegrable or maximal if there are $2 n-1$ functionally independent second-order constants of the motion:

$$
\begin{aligned}
\mathcal{L}_{\ell} & =\sum a^{j k}(\mathbf{q}) p_{j} p_{k}+W_{\ell}(\mathbf{q}) \\
\mathcal{L}_{0} & =\mathcal{H}, \quad \ell=0,1, \cdots, 2 n-2 \\
\left\{\mathcal{L}_{\ell}, \mathcal{H}\right\} & =0 .
\end{aligned}
$$

We say that the quantum system $H \Psi=E \Psi$ is superintegrable or maximal if there are $2 n-1$ linearly independent second-order symmetry operators:

$$
\begin{aligned}
L_{\ell} & =\sum \frac{1}{\sqrt{g}} \partial_{q_{j}}\left(\sqrt{g} a^{j k}(\mathbf{q}) \partial_{q_{k}}+W_{\ell}(\mathbf{q})\right. \\
L_{0} & =H, \quad \ell=0,1, \cdots, 2 n-2 \\
{\left[L_{\ell}, H\right] } & \equiv L_{\ell} H-H L_{\ell}=0 .
\end{aligned}
$$


To clarify the connection between these ideas and variable separation we assume that the coordinates $\mathbf{q}$ are orthogonal, i.e., the covariant metric tensor is diagonal:

$$
d s^{2}=\sum g_{j k} d q_{j} d q_{k}=\sum H_{j}^{2}(\mathbf{q}) d q_{j}^{2}
$$

so that the Hamilton-Jacobi equation is given by

$$
\mathcal{H}=\sum_{j} H_{j}^{-2}\left(\frac{\partial S}{\partial q_{j}}\right)^{2}+V(\mathbf{q})=E
$$

Set $\frac{\partial S}{\partial q_{j}}=S_{j}=p_{j}$ and assume additive separation in the $\mathbf{q}$ coordinates, so that $\partial_{j} S_{i}=\partial_{j} \partial_{i} S=0$ for $i \neq j$. The separation equations are postulated to be

$$
S_{i}^{2}-\sum_{j=1}^{n} u_{i j}\left(q_{i}\right) \lambda^{j}+f_{i}\left(q_{i}\right)=0, \quad i=1, \cdots, n, \quad \lambda^{1}=E .
$$

Here $\partial_{k} u_{i j}\left(q_{i}\right)=0$ for $k \neq i$ and $\operatorname{det}\left(u_{i j}\right) \neq 0$. We say that $U=\left(u_{i j}\right)$ is a Stäckel matrix.

Then (11) can be recovered from (12) provided $H_{j}^{-2}=\left(U^{-1}\right)^{1 j}$. The quadratic forms

$$
\mathcal{L}_{\ell}=\sum_{j=1}^{n}\left(U^{-1}\right)^{\ell j}\left(p_{j}^{2}+f_{j}\left(q_{j}\right)\right)=\sum_{j=1}^{n}\left(U^{-1}\right)^{\ell j} p_{j}^{2}+W_{\ell}(\mathbf{q})
$$

satisfy

$$
\mathcal{L}_{\ell}=-\lambda_{\ell}, \quad \mathcal{H}=\mathcal{L}_{1}=\sum H_{j}^{2} p_{j}^{2}+V(\mathbf{q})
$$

for a separable solution. Furthermore, we have

$$
\left\{\mathcal{L}_{\ell}, \mathcal{L}_{j}\right\}=0, \quad \ell \neq j
$$

Thus the $\mathcal{L}_{\ell}, \quad 2 \leq \ell \leq n$, are constants of the motion for the Hamiltonian $\mathcal{L}_{1}$.

An analogous construction, replacing (12) by $n$ second-order linear ODE's for factors $\Psi^{(i)}\left(q_{i}\right)$ leads to second order linear partial differential operators $L_{1}=H, L_{2}, \cdots, L_{n}$ such that

$$
H \Psi=E \Psi, \quad L_{\ell} \Psi=\lambda_{\ell} \Psi, \quad \ell=2, \cdots, n
$$


and

$$
\Psi(\mathbf{q})=\Pi_{k=1}^{n} \Psi^{(i)}\left(q_{i}\right)
$$

Then one can verify that

$$
\left[L_{k}, H\right]=0, \quad\left[L_{k}, L_{j}\right]=0 .
$$

How does one find all orthogonal separable coordinate systems $\mathbf{q}$ for a given space $R_{n}$ for zero potential, $V \equiv 0$ ? This is a difficult problem in differential geometry. The answer is known for some constant curvature spaces. In real Euclidean 2-space there are four separable systems: cartesian, polar, parabolic and elliptic. For complex Euclidean 2-space, including real Euclidean space and real Minkowski space, there are six $[4,16,18]$ : Cartesian, polar, parabolic, elliptic, hyperbolic and semi-hyperbolic. We describe these coordinate systems and their corresponding free particle constants of the motion $L$. (We adopt the basis $p_{x}, p_{y}, M=x p_{y}-y p_{x}$ for the Lie algebra $e(2, C)$ and define $p_{ \pm}=p_{x} \pm i p_{y}, \smile$.) There is one orbit of constants of the motion, with representative $M p_{+}$, that is not associated with variable separation [21]. The separable systems are:

\section{Cartesian coordinates}

$$
x, y, \quad L=p_{x}^{2}
$$

\section{Polar Coordinates}

$$
\begin{aligned}
& x=r \cos \theta, \\
& y=r \sin \theta, \quad L=M^{2}
\end{aligned}
$$

Parabolic Coordinates.

$$
x_{P}=\frac{1}{2}\left(\xi^{2}-\eta^{2}\right), \quad y_{P}=\xi \eta, \quad L=M p_{y}
$$

Elliptic Coordinates (in algebraic form)

$$
\begin{aligned}
x_{E}^{2} & =c^{2}(u-1)(v-1), \quad y_{E}^{2}=-c^{2} u v \\
L & =M^{2}+c^{2} p_{x}^{2}
\end{aligned}
$$

\section{Hyperbolic Coordinates}

$$
\begin{aligned}
x_{H} & =\frac{r^{2}+r^{2} s^{2}+s^{2}}{2 r s}, \quad y_{H}=i \frac{r^{2}-r^{2} s^{2}+s^{2}}{2 r s}, \\
L & =M^{2}+p_{+}^{2}
\end{aligned}
$$




\section{Semi-Hyperbolic Coordinates}

$$
\begin{aligned}
x_{S H} & =-\frac{1}{4}(w-u)^{2}+\frac{1}{2}(w+u), \quad i y_{S H}=-\frac{1}{4}(w-u)^{2}-\frac{1}{2}(w+u)(19) \\
L & =2 M p_{+}+p_{-}^{2}
\end{aligned}
$$

In real Euclidean 3-space there are 11 separable systems see Table 1, $[4,16]$. On the real 2-sphere there are 2: spherical and ellipsoidal. For real $n$-dimensional Euclidean space and the $n$-sphere Kalnins and the author have a graphical procedure to classify and construct all possibilities, $[17,18]$. On the 2-hyperboloid there are 9 separable systems, [18]. For the $n$-hyperboloid of two sheets there is again a graphical procedure to construct all possibilites, [18].

In each case above, the symmetries $\mathcal{L}_{j}$ are second order elements in the enveloping algebra of the symmetry Lie algebra of the corresponding manifold, e.g., the Lie algebra $e(n, C)$ for Euclidean $n$-space and $s o(n+1, C)$ for the $n$-sphere, $\smile$.

We see that for zero potential, each of the constant curvature spaces listed above is separable in multiple coordinate systems. Indeed we can veryfy that the zero potential is superintegrable on each of these spaces. However, a potential $V \neq 0$ "breaks the symmetry" and reduces the number of separable systems, usually to zero. (See [21] for conditions that must be satisfied by a potential in order to permit separation in a given coordinate system.)

How does one determine which constants of the motion lead to variable separation?

Theorem 1 Necessary and sufficient conditions for the existence of an orthogonal separable coordinate system $\left\{q_{i}\right\}$ for the Hamilton-Jacobi equation $\mathcal{H}^{1}=E$ on an $n$-dimensional Riemannian manifold are that there exist $n$ quadratic forms $\mathcal{L}_{k}=\sum_{i, j=1}^{n} L_{i j}^{(k)} p_{i} p_{j}+W_{k}$ on the manifold such that:

1. $\left\{\mathcal{L}_{k}, \mathcal{L}_{\ell}\right\}=0, \quad 1 \leq k, i \leq n$,

2. The set $\left\{\mathcal{L}_{k}\right\}$ is linearly independent (as $n$ quadratic forms).

3. There is a basis $\left\{\omega_{(j)}: 1 \leq j \leq n\right\}$ of simultaneous eigenforms for the $n$ matrices $\left\{L_{i j}^{(k)}\right\}$. 


\begin{tabular}{|c|c|}
\hline Table 1 Separable coordinates & in 3-D real Euclidean space. \\
\hline Coordinate System & Coordinates \\
\hline $\begin{array}{l}\text { I. Cartesian } \\
x, y, z \in \mathbf{R}\end{array}$ & $x, y, z$ \\
\hline $\begin{array}{l}\text { II. Cylindrical polar } \\
\rho>0, \varphi \in[0,2 \pi)\end{array}$ & $x=\rho \cos \varphi, y=\rho \sin \varphi, z$ \\
\hline $\begin{array}{l}\text { III. Cylindrical elliptic } \\
z \in \mathbf{R}, e_{1}<\mu_{1}<e_{2}<\mu_{2}\end{array}$ & $x^{2}=\frac{\left(\mu_{1}-e_{1}\right)\left(\mu_{2}-e_{1}\right)}{\left(e_{2}-e_{1}\right)}, \quad y^{2}=\frac{\left(\mu_{1}-e_{2}\right)\left(\mu_{2}-e_{2}\right)}{\left(e_{1}-e_{2}\right)}, z$ \\
\hline $\begin{array}{l}\text { IV. Cylindrical parabolic } \\
\xi, x \in \mathbf{R}, \eta \geq 0\end{array}$ & $x, y=\xi \eta, \quad z=\frac{1}{2}\left(\xi^{2}-\eta^{2}\right)$ \\
\hline $\begin{array}{l}\text { V. Spherical } \\
r>0, \theta \in[0, \pi], \quad \varphi \in[0,2 \pi)\end{array}$ & $x=r \cos \theta \cos \varphi, \quad y=r \sin \theta \sin \varphi, \quad z=r \cos \theta$ \\
\hline $\begin{array}{l}\text { VI. Prolate spheroidal } \\
e_{1}<u_{1}<e_{2}<u_{2} \\
\varphi \in[0,2 \pi)\end{array}$ & $\begin{array}{l}x^{2}=\frac{\left(u_{1}-e_{2}\right)\left(u_{2}-e_{2}\right)}{\left(e_{1}-e_{2}\right)} \cos ^{2} \varphi, y^{2}=\frac{\left(u_{1}-e_{2}\right)\left(u_{2}-e_{2}\right)}{\left(e_{1}-e_{2}\right)} \sin ^{2} \varphi \\
z^{2}=\frac{\left(u_{1}-e_{1}\right)\left(u_{2}-e_{1}\right)}{\left(e_{2}-e_{1}\right)}\end{array}$ \\
\hline $\begin{array}{l}\text { VII. Oblate spheroidal } \\
e_{1}<u_{1}<e_{2}<u_{2} \\
\varphi \in[0,2 \pi)\end{array}$ & $\begin{array}{l}x^{2}=\frac{\left(u_{1}-e_{1}\right)\left(u_{2}-e_{1}\right)}{\left(e_{2}-e_{1}\right)} \cos ^{2} \varphi, \quad y^{2}=\frac{\left(u_{1}-e_{1}\right)\left(u_{2}-e_{1}\right)}{\left(e_{2}-e_{1}\right)} \sin ^{2} \varphi \\
z^{2}=\frac{\left(u_{1}-e_{2}\right)\left(u_{2}-e_{2}\right)}{\left(e_{1}-e_{2}\right)}\end{array}$ \\
\hline $\begin{array}{l}\text { VIII. Sphero-conical } \\
r \geq 0 \\
e_{1}<\rho_{1}<e_{2}<\rho_{2}<e_{3}\end{array}$ & $\begin{array}{l}x^{2}=r^{2} \frac{\left(\rho_{1}-e_{1}\right)\left(\rho_{2}-e_{1}\right)}{\left(e_{1}-e_{2}\right)\left(e_{1}-e_{3}\right)}, \quad y^{2}=r^{2} \frac{\left(\rho_{1}-e_{2}\right)\left(\rho_{2}-e_{2}\right)}{\left(e_{2}-e_{1}\right)\left(e_{2}-e_{3}\right)} \\
z^{2}=r^{2} \frac{\left(\rho_{1}-e_{3}\right)\left(\rho_{2}-e_{3}\right)}{\left(e_{3}-e_{2}\right)\left(e_{3}-e_{1}\right)}\end{array}$ \\
\hline $\begin{array}{l}\text { IX. Parabolic } \\
\xi, \eta \geq 0, \quad \varphi \in[0,2 \pi)\end{array}$ & $x=\xi \eta \cos \varphi, \quad y=\xi \eta \sin \varphi, \quad z=\frac{1}{2}\left(\xi^{2}-\eta^{2}\right)$ \\
\hline $\begin{array}{l}\text { X. Ellipsoidal } \\
a_{1}<u_{1}<a_{2}<u_{2}<a_{3}<u_{3}\end{array}$ & $\begin{array}{l}x^{2}=\frac{\left(u_{1}-a_{1}\right)\left(u_{2}-a_{1}\right)\left(u_{3}-a_{1}\right)}{\left(a_{3}-a_{1}\right)\left(a_{2}-a_{1}\right)}, y^{2}=\frac{\left(u_{1}-a_{2}\right)\left(u_{2}-a_{2}\right)\left(u_{3}-a_{2}\right)}{\left(a_{1}-a_{2}\right)\left(a_{3}-a_{2}\right)} \\
z^{2}=\frac{\left(u_{1}-a_{3}\right)\left(u_{2}-a_{3}\right)\left(u_{3}-a_{3}\right)}{\left(a_{1}-a_{3}\right)\left(a_{2}-a_{3}\right)}\end{array}$ \\
\hline $\begin{array}{l}\text { XI. Paraboloidal } \\
0<\eta_{1}<a_{2}<\eta_{2}<a_{3}<\eta_{3}\end{array}$ & $\begin{array}{l}x^{2}=\frac{\left(\eta_{1}-a_{3}\right)\left(\eta_{2}-a_{3}\right)\left(\eta_{3}-a_{3}\right)}{\left(a_{3}-a_{2}\right)}, y^{2}=\frac{\left(\eta_{1}-a_{2}\right)\left(\eta_{2}-a_{2}\right)\left(\eta_{3}-a_{2}\right)}{\left(a_{2}-a_{3}\right)} \\
z^{2}=\frac{1}{2}\left(\eta_{1}+\eta_{2}+\eta_{3}-a_{2}-a_{3}\right)\end{array}$ \\
\hline
\end{tabular}


If conditions (1)-(3) are satisfied then there exist functions $g_{i}(\mathbf{q})$ such that:

$$
\omega_{(j)}=g_{j} d q_{j}, \quad j=1, \cdots, n .
$$

Theorem 2 Necessary and sufficient conditions for the existence of an orthogonal separable coordinate system $\left\{q_{i}\right\}$ for the Schrödinger equation $\left(\Delta_{n}+\right.$ $V) \Psi=E \Psi$ on an n-dimensional constant curvature space are that there exists a linearly independent set $\left\{L_{1}=H=\Delta_{n}+V, L_{2}, \cdots, L_{n}\right\}$ of second-order differential operators on the manifold such that:

1. $\left[L_{k}, L_{\ell}\right]=0, \quad 1 \leq k, \ell \leq n$,

2. Each $L_{k}$ is in self-adjoint form,

3. There is a basis $\left\{\omega_{(j)}: 1 \leq j \leq n\right\}$ of simultaneous eigenforms for the $\left\{L_{k}\right\}$.

If conditions (1)-(3) are satisfied then there exist functions $g_{i}(\mathbf{q})$ such that:

$$
\omega_{(j)}=g_{j} d q_{j}, \quad j=1, \cdots, n .
$$

See $[28,21]$ for proofs and discussions of these theorems. The main point of the theorems is that, under the required hypotheses the eigenforms $\omega^{\ell}$ of the quadratic forms $L^{i j}$ are normalizable, i.e., that up to multiplication by a nonzero function, $\omega^{\ell}$ is the differential of a coordinate. This fact permits us to compute the coordinates directly from a knowledge of the symmetry operators. For general Riemannian manifolds Theorem 1 remains true, but Theorem 2 is false unless separation is replaced by the more general concept of R-separation [21].

We expect a superintegrable system to separate in multiple coordinate systems, though the above remarks do not constitute a proof of this. Thus, one way to find superintegrable systems is to search for potentials $V(\mathbf{q})$ that permit separation in multiple coordinate systems. 


\section{Examples for the Euclidean plane}

To illustrate the basic ideas we can consider the example of the Schrödinger equation with potential

$$
V(x, y)=\frac{1}{2}\left(\omega^{2}\left(x^{2}+y^{2}\right)+\frac{k_{1}^{2}-\frac{1}{4}}{x^{2}}+\frac{k_{2}^{2}-\frac{1}{4}}{y^{2}}\right),
$$

i.e.,

$$
\left(\frac{\partial^{2}}{\partial x^{2}}+\frac{\partial^{2}}{\partial y^{2}}\right) \Psi-\left(\omega^{2}\left(x^{2}+y^{2}\right)+\frac{k_{1}^{2}-\frac{1}{4}}{x^{2}}+\frac{k_{2}^{2}-\frac{1}{4}}{y^{2}}\right) \Psi=-2 E \Psi .
$$

This equation separates in three coordinate systems: Cartesian coordinates $(x, y)$; polar coordinates $x=r \cos \theta, y=r \sin \theta$, and elliptical coordinates

$$
x^{2}=c^{2} \frac{\left(u_{1}-e_{1}\right)\left(u_{2}-e_{1}\right)}{\left(e_{1}-e_{2}\right)}, \quad y^{2}=c^{2} \frac{\left(u_{1}-e_{2}\right)\left(u_{2}-e_{2}\right)}{\left(e_{2}-e_{1}\right)} .
$$

The bound state energies are given by

$$
E_{n}=\omega\left(2 n+2+k_{1}+k_{2}\right)
$$

for integer $n$. The wave functions for each of these coordinate systems are: 1. Cartesian coordinates

$$
\begin{gathered}
\Psi_{n_{1}, n_{2}}(x, y)=2 \omega^{\frac{1}{2}\left(k_{1}+k_{2}+2\right)} \sqrt{\frac{n_{1} ! n_{2} !}{\Gamma\left(n_{1}+k_{1}+1\right) \Gamma\left(n_{2}+k_{2}+1\right)}} x^{\left(k_{1}+\frac{1}{2}\right)} y^{\left(k_{2}+\frac{1}{2}\right)} \\
e^{-\frac{\omega}{2}\left(x^{2}+y^{2}\right)} L_{n_{1}}^{k_{1}}\left(\omega x^{2}\right) L_{n_{2}}^{k_{2}}\left(\omega y^{2}\right)
\end{gathered}
$$

where $n=n_{1}+n_{2}$, and the $L_{n}^{k}(x)$ are Laguerre polynomials, [29]

2. polar coordinates

$$
\begin{gathered}
\Psi(r, \theta)=\Phi_{q}^{\left(k_{1}, k_{2}\right)}(\theta) \omega^{\frac{1}{2}\left(2 q+k_{1}+k_{2}+1\right)} \sqrt{\frac{2 m !}{\Gamma\left(m+2 q+k_{1}+k_{2}+1\right)}} \\
e^{\left(-\omega r^{2} / 2\right)} r^{\left(2 q+k_{1}+k_{2}+1\right)} L_{m}^{2 q+k_{1}+k_{2}+1}\left(\omega r^{2}\right)
\end{gathered}
$$

where $n=m+q$,

$$
\Phi_{q}^{\left(k_{1}, k_{2}\right)}(\theta)=\sqrt{2\left(2 q+k_{1}+k_{2}+1\right) \frac{q ! \Gamma\left(k_{1}+k_{2}+q+1\right)}{\Gamma\left(k_{2}+q+1\right) \Gamma\left(k_{1}+q+1\right)}}
$$




$$
\times(\cos \theta)^{k_{1}+(1 / 2)}(\sin \theta)^{k_{2}+(1 / 2)} P_{q}^{\left(k_{1}, k_{2}\right)}(\cos 2 \theta),
$$

and the $P_{q}^{\left(k_{1}, k_{2}\right)}(\cos 2 \theta)$ are Jacobi polynomials, [29].

3. elliptical coordinates

$$
\Psi=e^{-\omega\left(x^{2}+y^{2}\right)} x^{k_{1}+\frac{1}{2}} y^{k_{2}+\frac{1}{2}} \prod_{m=1}^{n}\left(\frac{x^{2}}{\theta_{m}-e_{1}}+\frac{y^{2}}{\theta_{m}-e_{2}}-c^{2}\right)
$$

where use has been made of the identity

$$
\frac{x^{2}}{\theta-e_{1}}+\frac{y^{2}}{\theta-e_{2}}-c^{2}=-c^{2} \frac{\left(u_{1}-\theta\right)\left(u_{2}-\theta\right)}{\left(\theta-e_{1}\right)\left(\theta-e_{2}\right)} .
$$

The zeros $\theta_{j}$ satisfy the relations

$$
\frac{k_{1}+1}{\theta_{m}-e_{1}}+\frac{k_{2}+1}{\theta_{m}-e_{2}}+\sum_{j \neq m} \frac{2}{\left(\theta_{m}-\theta_{j}\right)}-\omega=0 .
$$

Associated with the separability of the Schrödinger equation in these coordinate systems there are second order symmetry operators. A basis for such operators is

$$
\begin{gathered}
L_{1}=\partial_{x}^{2}+\frac{\left(\frac{1}{4}-k_{1}^{2}\right)}{x^{2}}-\omega^{2} x^{2}, \quad L_{2}=\partial_{y}^{2}+\frac{\left(\frac{1}{4}-k_{2}^{2}\right)}{y^{2}}-\omega^{2} y^{2} \\
M=\left(x \partial_{y}-y \partial_{x}\right)^{2}+\left(\frac{1}{4}-k_{1}^{2}\right) \frac{y^{2}}{x^{2}}+\left(\frac{1}{4}-k_{2}^{2}\right) \frac{x^{2}}{y^{2}}-\frac{1}{2} .
\end{gathered}
$$

(Note that $H=L_{1}+L_{2}$.) The separable eigenfunctions already given are eigenfunctions of the symmetry operators $L_{1}, M$ and $M+e_{2} L_{1}+e_{1} L_{2}$ with eigenvalues

$$
\begin{gathered}
\lambda_{c}=-\omega\left(2 n_{1}+k_{1}+1\right), \quad \lambda_{p}=\left(2 q+k_{1}+k_{2}+1\right)^{2}+\left(1+k_{1}^{2}+k_{2}^{2}\right), \\
\lambda_{e}=2\left(1-k_{1}\right)\left(1-k_{2}\right)-2 e_{2} \omega\left(k_{1}+1\right)-2 e_{1} \omega\left(k_{2}+1\right)-\omega^{2} e_{1} e_{2}- \\
4 \sum_{m=1}^{q}\left[e_{2} \frac{k_{1}+1}{\theta_{m}-e_{1}}+e_{1} \frac{k_{2}+1}{\theta_{m}-e_{2}}\right] .
\end{gathered}
$$

The algebra constructed by repeated commutators is ( $R$ is defined by the first relation)

$$
\left[L_{1}, M\right]=\left[M, L_{2}\right]=R, \quad\left[L_{i}, R\right]=-4\left\{L_{i}, L_{j}\right\}+16 \omega^{2} M, \quad i \neq j
$$




$$
\begin{aligned}
& {[M, R]=4\left\{L_{1}, M\right\}-4\left\{L_{2}, M\right\}+8\left(1-k_{2}^{2}\right) L_{1}-8\left(1-k_{1}^{2}\right) L_{2},} \\
& R^{2}=\frac{8}{3}\left\{M, L_{1}, L_{2}\right\}+\frac{64}{3}\left\{L_{1}, L_{2}\right\}+16 \omega^{2} M^{2}-16\left(1-k_{2}^{2}\right) L_{1}^{2} \\
& \quad-16\left(1-k_{1}^{2}\right) L_{2}^{2}-\frac{128}{3} \omega^{2} M-64 \omega^{2}\left(1-k_{1}^{2}\right)\left(1-k_{2}^{2}\right) .
\end{aligned}
$$

These relations are quadratic.

In real Euclidean two-space there are precisely four potentials that have the multiseparation property, [14]. The second potential is

$$
V(x, y)=\omega^{2}\left(4 x^{2}+y^{2}\right)-\frac{\left(\frac{1}{4}-k_{2}^{2}\right)}{y^{2}} .
$$

The corresponding Schrödinger equation is separable in two coordinate systems: Cartesian coordinates and parabolic coordinates

$$
x=\frac{1}{2}\left(\xi^{2}-\eta^{2}\right), y=\xi \eta .
$$

The third potential is

$$
V(x, y)=-\frac{\alpha}{\sqrt{x^{2}+y^{2}}}+\frac{1}{4 \sqrt{x^{2}+y^{2}}}\left(\frac{\left(k_{1}^{2}-\frac{1}{4}\right)}{\sqrt{x^{2}+y^{2}}+x}+\frac{\left(k_{2}^{2}-\frac{1}{4}\right)}{\sqrt{x^{2}+y^{2}}-x}\right) .
$$

The corresponding Schrödinger equation is separable in two coordinate systems: polar, parabolic and modified elliptic coordinates, where

$$
\xi^{2}=c^{2} \frac{\left(u_{1}-e_{1}\right)\left(u_{2}-e_{1}\right)}{\left(e_{1}-e_{2}\right)}, \quad \eta^{2}=c^{2} \frac{\left(u_{1}-e_{2}\right)\left(u_{2}-e_{2}\right)}{\left(e_{2}-e_{1}\right)} .
$$

This last coordinate system can be written as

$$
x=\sqrt{\frac{\left(U_{1}-E_{1}\right)\left(U_{2}-E_{1}\right)}{4\left(E_{1}-E_{2}\right)}}, \quad y=\sqrt{\frac{\left(U_{1}-E_{2}\right)\left(U_{2}-E_{2}\right)}{4\left(E_{2}-E_{1}\right)}}-2 \sqrt{E_{1}-E_{2}},
$$

where $E_{1}=-e_{1} e_{2}, E_{2}=-\frac{1}{4}\left(e_{1}+e_{2}\right)^{2}$ and $U_{j}=u_{j}^{2}-u_{j}\left(e_{1}+e_{2}\right)$.

The fourth potential is

$$
V(x, y)=-\frac{\alpha}{\sqrt{x^{2}+y^{2}}}+\frac{B_{1}}{4} \frac{\sqrt{\sqrt{x^{2}+y^{2}}+x}}{\sqrt{x^{2}+y^{2}}}+\frac{B_{2}}{4} \frac{\sqrt{\sqrt{x^{2}+y^{2}}-x}}{\sqrt{x^{2}+y^{2}}} .
$$


Separation occurs here in parabolic and parabolic coordinates of the second type

$$
x=\mu \nu, \quad y=\frac{1}{2}\left(\mu^{2}-\nu^{2}\right) .
$$

As an illustration of the utility of the notion of a quadratic algebra consider the last potential given. A basis for the quadratic algebra consists of $L_{1}, L_{2}$ and $H$ with defining relations

$$
\begin{gathered}
{\left[R, L_{1}\right]=-4 L_{2} H+B_{1} B_{2}, \quad\left[R, L_{2}\right]=4 L_{1} H+\frac{1}{2}\left(B_{1}^{2}-B_{2}^{2}\right)} \\
R^{2}=4 L_{1}^{2} H+4 L_{2}^{2} H-16 \alpha^{2} H+\left(B_{2}^{2}-B_{1}^{2}\right) L_{1}-2 B_{1} B_{2} L_{2}-2 \alpha^{2}\left(B_{1}^{2}+B_{2}^{2}\right)
\end{gathered}
$$

with $R=\left[L_{1}, L_{2}\right]$. If we look for eigenfunctions of the operators $L_{1}, L_{2}$ respectively, we have

$$
L_{1} \varphi_{m}=\lambda_{m} \varphi_{m}, \quad L_{2} \psi_{n}=\rho_{n} \psi_{n} .
$$

If we write

$$
L_{1} \psi_{n}=\sum_{\tau} C_{n \tau} \psi_{\tau}
$$

then the quadratic algebra relations imply

$$
\begin{gathered}
{\left[\left(\rho_{n}-\rho_{\tau}\right)^{2}+8 E\right] C_{n \tau}=-\left[\frac{1}{2}\left(B_{1}^{2}-B_{2}^{2}\right)-16 \alpha E\right] \delta_{n \tau}} \\
\sum_{\tau} C_{n \tau} C_{\tau \sigma}\left(2 \rho_{\tau}-\rho_{n}-\rho_{\sigma}\right)=\left(8 E \rho_{n}+B_{1} B_{2}+16 \alpha E\right) \delta_{n \sigma} .
\end{gathered}
$$

These relations in turn imply that

$$
C_{n n}=-\frac{\frac{1}{2}\left(B_{1}^{2}-B_{2}^{2}\right)+16 \alpha E}{8 E}
$$

and $C_{n n+1}=C_{n+1 n}^{*}$ are the only nonzero coefficents. Indeed they can essentially be determined by the relation

$$
4 \sqrt{-2 E}\left(\left|C_{n, n+1}\right|^{2}-\left|C_{n-1, n}\right|^{2}\right)=8 E \rho_{n}+B_{1} B_{2}+16 \alpha E
$$

where the eigenvalues $\lambda_{m}$ and $\rho_{n}$ are given by

$\lambda_{m}=2 \alpha-\frac{B_{1}^{2}}{8 E}-(2 m+1) \sqrt{-2 E}, \quad \rho_{n}=2 \alpha-\frac{\left(B_{1}+B_{2}\right)^{2}}{16 E}-(2 n+1) \sqrt{-2 E}$

and the quantisation condition for $E$ is

$$
4 \alpha-\frac{B_{1}^{2}+B_{2}^{2}}{8 E}=-(q+2) \sqrt{-2 E}
$$

for integer $q$. 


\section{More examples: The real 2-sphere}

These ideas work also for separable coordinates on the real two dimensional sphere, [14]. An important example is The potential

$$
V=\frac{1}{2}\left[\frac{k_{1}^{2}-\frac{1}{4}}{s_{1}^{2}}+\frac{k_{2}^{2}-\frac{1}{4}}{s_{2}^{2}}+\frac{k_{3}^{2}-\frac{1}{4}}{s_{3}^{2}}\right]
$$

where $s_{1}^{2}+s_{2}^{2}+s_{3}^{2}=1$. The corresponding Schrödinger equation has the form

$$
\begin{gathered}
{\left[\left(s_{1} \frac{\partial}{\partial s_{2}}-s_{2} \frac{\partial}{\partial s_{1}}\right)^{2}+\left(s_{1} \frac{\partial}{\partial s_{3}}-s_{3} \frac{\partial}{\partial s_{1}}\right)^{2}+\left(s_{3} \frac{\partial}{\partial s_{2}}-s_{2} \frac{\partial}{\partial s_{3}}\right)^{2}\right] \Psi+} \\
{\left[\frac{\left(k_{1}^{2}-\frac{1}{4}\right)}{s_{1}^{2}}+\frac{\left(k_{2}^{2}-\frac{1}{4}\right)}{s_{2}^{2}}+\frac{\left(k_{3}^{2}-\frac{1}{4}\right)}{s_{3}^{2}}\right] \Psi=-2 E \Psi .}
\end{gathered}
$$

This equation admits solution via separation of variables in two coordinate systems: spherical coordinates

$$
s_{1}=\sin \theta \cos \varphi, s_{2}=\sin \theta \sin \varphi, s_{3}=\cos \theta
$$

and elliptical coordinates

$$
s_{i}^{2}=\frac{\left(u_{1}-e_{i}\right)\left(u_{2}-e_{i}\right)}{\left(e_{1}-e_{j}\right)\left(e_{i}-e_{j}\right)}, \quad i, j, k=1,2,3 \quad i \neq j \neq k .
$$

Indeed a basis for the second-order symmetries of Schrödinger's equation with this potential is

$$
L_{i j}=\left(s_{i} \partial_{s_{j}}-s_{j} \partial_{s_{i}}\right)^{2}+\left(\frac{1}{4}-k_{i}^{2}\right) \frac{s_{j}^{2}}{s_{i}^{2}}+\left(\frac{1}{4}-k_{j}^{2}\right) \frac{s_{i}^{2}}{s_{j}^{2}}-\frac{1}{2}, \quad i \neq j
$$

These symmetries satisfy the quadratic algebra relations

$$
\begin{gathered}
{\left[L_{i j}, R\right]=4\left\{L_{i j}, L_{j k}\right\}-4\left\{L_{i j}, L_{i k}\right\}+8\left(1-k_{i}^{2}\right) L_{j k}-8\left(1-k_{j}\right)^{2} L_{i k},} \\
R^{2}=-\frac{4}{3}\left\{L_{i j}, L_{i k}, L_{j k}\right\}+\frac{64}{3}\left\{L_{i j}, L_{i k}\right\}+\frac{64}{3}\left\{L_{i j}, L_{j k}\right\}+\frac{64}{3}\left\{L_{i k}, L_{j k}\right\}- \\
16\left(1-k_{k}^{2}\right) L_{i j}^{2}-16\left(1-k_{j}^{2}\right) L_{i k}^{2}-16\left(1-k_{i}^{2}\right) L_{j k}^{2}+
\end{gathered}
$$




$$
\frac{128}{3}\left(1-k_{i}^{2}\right) L_{j k}+\frac{128}{3}\left(1-k_{j}^{2}\right) L_{i k}+\frac{128}{3}\left(1-k_{k}^{2}\right) L_{i j}, \quad i \neq j \neq k .
$$

The eigenfunctions with bound state energy eigenvalues

$$
E_{p}=\frac{1}{2}\left(2 p+2+k_{1}+k_{2}+k_{3}\right)^{2}-\frac{1}{8}
$$

in these coordinate systems are: polar

$$
\Psi=(\sin \theta)^{-1} \Phi_{n}^{\left(k_{2}, k_{1}\right)}(\varphi) \Phi_{m}^{\left(2 n+k_{1}+k_{2}, k_{3}\right)}(\theta)
$$

where $p=m+n,\left(\right.$ here $\Phi_{n}^{\left(k_{2}, k_{1}\right)}$ is given by (20)) and elliptical

$$
\Psi=\left(\prod_{\ell=1}^{3} s_{\ell}^{\left(k_{\ell}+\frac{1}{2}\right)}\right) \prod_{j=1}^{q}\left(\frac{s_{1}^{2}}{\theta_{j}-e_{1}}+\frac{s_{2}^{2}}{\theta_{j}-e_{2}}+\frac{s_{3}^{2}}{\theta_{j}-e_{3}}\right)
$$

where

$$
\frac{k_{1}+1}{\theta_{m}-e_{1}}+\frac{k_{2}+1}{\theta_{m}-e_{2}}+\frac{k_{3}+1}{\theta_{m}-e_{3}}+\sum_{j \neq m} \frac{2}{\left(\theta_{m}-\theta_{j}\right)}=0
$$

and $q=p$. Here we have made use of the identity

$$
\frac{s_{1}^{2}}{\theta_{j}-e_{1}}+\frac{s_{2}^{2}}{\theta_{j}-e_{2}}+\frac{s_{3}^{2}}{\theta_{j}-e_{3}}=\frac{\Pi_{\ell=1}^{2}\left(u_{\ell}-\theta_{j}\right)}{\Pi_{m=1}^{3}\left(\theta_{j}-e_{m}\right)} .
$$

The separable eigenfunctions already given are eigenfunctions of the symmetry operators $L_{12}$ and $e_{3} L_{12}+e_{2} L_{13}+e_{1} L_{23}$ :

$$
\begin{gathered}
\lambda_{S}=\left(2 n+k_{1}+k_{2}+1\right)^{2}, \\
\lambda_{E}=-2\left[k_{1}\left(e_{2}+e_{3}\right)+k_{2}\left(e_{1}+e_{3}\right)+k_{3}\left(e_{2}+e_{1}\right)+e_{3} k_{1} k_{2}+e_{1} k_{2} k_{3}+e_{2} k_{1} k_{3}\right]- \\
\frac{3}{2}\left(e_{1}+e_{2}+e_{3}\right)-4 e_{2} e_{3}\left(k_{1}+1\right) \sum_{m=1}^{q} \frac{1}{\theta_{m}-e_{1}}- \\
4 e_{2} e_{1}\left(k_{3}+1\right) \sum_{m=1}^{q} \frac{1}{\theta_{m}-e_{3}}-4 e_{1} e_{3}\left(k_{2}+1\right) \sum_{m=1}^{q} \frac{1}{\theta_{m}-e_{2}} .
\end{gathered}
$$

A second multiseparable potential on the sphere is

$$
V=-\frac{\alpha s_{3}}{\sqrt{s_{1}^{2}+s_{2}^{2}}}+\frac{1}{4 \sqrt{s_{1}^{2}+s_{2}^{2}}}\left[\frac{\left(k_{1}^{2}-\frac{1}{4}\right)}{\sqrt{s_{1}^{2}+s_{2}^{2}}+s_{1}}+\frac{\left(k_{2}^{2}-\frac{1}{4}\right)}{\sqrt{s_{1}^{2}+s_{2}^{2}}-s_{1}}\right]
$$


Schrödinger's equation for this potential has the form

$$
\begin{gathered}
{\left[\left(s_{1} \frac{\partial}{\partial s_{2}}-s_{2} \frac{\partial}{\partial s_{1}}\right)^{2}+\left(s_{1} \frac{\partial}{\partial s_{3}}-s_{3} \frac{\partial}{\partial s_{1}}\right)^{2}+\left(s_{3} \frac{\partial}{\partial s_{2}}-s_{2} \frac{\partial}{\partial s_{3}}\right)^{2}\right] \Psi+} \\
\left(-\frac{\alpha s_{3}}{\sqrt{s_{1}^{2}+s_{2}^{2}}}+\frac{1}{4 \sqrt{s_{1}^{2}+s_{2}^{2}}}\left[\frac{\left(k_{1}^{2}-\frac{1}{4}\right)}{\sqrt{s_{1}^{2}+s_{2}^{2}}+s_{1}}+\frac{\left(k_{2}^{2}-\frac{1}{4}\right)}{\sqrt{s_{1}^{2}+s_{2}^{2}}-s_{1}}\right]\right) \Psi=-2 E \Psi .
\end{gathered}
$$

This equation admits solution via separation of variables in two coordinates systems: spherical and elliptical coordinates of modified type

$$
s_{1}^{\prime}=\cos f s_{1}+\sin f s_{3}, \quad s_{2}^{\prime}=s_{2}, \quad s_{3}^{\prime}=-\sin f s_{1}+\cos f s_{3}
$$

where

$$
s_{i}^{2}=\frac{\left(y_{1}-e_{i}\right)\left(y_{2}-e_{i}\right)}{\left(e_{1}-e_{j}\right)\left(e_{i}-e_{j}\right)}, \quad i, j, k=1,2,3 \quad i \neq j \neq k
$$

and

$$
\begin{gathered}
\sin f=\sqrt{\frac{\left(e_{2}-e_{1}\right)}{\left(e_{3}-e_{1}\right)}}, \quad e_{1}=e_{2}+\frac{1}{4}\left(E_{+}+E_{-}\right)^{2}, \quad e_{3}=e_{2}+\frac{1}{4}\left(E_{+}-E_{-}\right)^{2}, \\
y_{j}=e_{2}+\frac{1}{4}\left(E_{+}^{2}+E_{-}^{2}\right)+\frac{1}{4} E_{+} E_{-}\left(Z_{j}+\frac{1}{Z_{j}}\right), \quad j=1,2 .
\end{gathered}
$$

Indeed if we use the variables

$$
U_{3}^{2}=Z_{1} Z_{2}, \quad U_{1}^{2}=\frac{\left(Z_{1}+\Omega_{-}\right)\left(Z_{2}+\Omega_{-}\right)}{\left(\Omega_{-}^{2}-1\right)}, \quad U_{2}^{2}=\frac{\left(Z_{1}+\Omega_{+}\right)\left(Z_{2}+\Omega_{+}\right)}{\left(\Omega_{+}^{2}-1\right)}
$$

where

$$
\Omega_{+}=\frac{E_{+}}{E_{-}}, \Omega_{-}=\frac{E_{-}}{E_{+}}
$$

then, putting $k_{3}=\sqrt{2(E-i \alpha)+1 / 4}$ and $\hat{E}=i \alpha+E$ and multiplying the Schrödinger equation by $\left(Z_{1} Z_{2}\right)^{-1}-1$, we see that the resulting equation has the form

$$
\left.H \Psi=\left(U_{1} \frac{\partial}{\partial U_{2}}-U_{2} \frac{\partial}{\partial U_{1}}\right)^{2}+\left(U_{1} \frac{\partial}{\partial U_{3}}-U_{3} \frac{\partial}{\partial U_{1}}\right)^{2}+\left(U_{3} \frac{\partial}{\partial U_{2}}-U_{2} \frac{\partial}{\partial U_{3}}\right)^{2}\right) \Psi+
$$




$$
\left[\frac{\left(\frac{1}{4}-k_{1}^{2}\right)}{U_{1}^{2}}+\frac{\left(\frac{1}{4}-k_{2}^{2}\right)}{U_{2}^{2}}+\frac{\left(\frac{1}{4}-k_{3}^{2}\right)}{U_{3}^{2}}\right] \Psi+2 \hat{E} \Psi=0
$$

which is essentially the same form as for the first potential. The bound state quantisation condition has the form

$$
E=\frac{1}{2}\left[2 q+2+k_{1}+k_{2}+\sqrt{\frac{1}{4}+2(E-i \alpha)}\right]^{2}-\frac{1}{8} .
$$

\section{Features of superintegrability $(n=2)$}

Based on the examples of the last two sections, we can point out some basic features of superintegrability in two dimensions.

1. The potential $V$ permits separability of the Hamilton-Jacobi equation $\mathcal{H}=E$ and the Schrödinger equation $H \Psi=E \Psi$ in at least two coordinate systems, characterized by symmetry conditions $\mathcal{L}_{1}=\lambda_{1}, \mathcal{L}_{2}=\lambda_{2}$ in the first case and $L_{1} \Psi=\lambda_{1} \Psi, L_{2} \Psi=\lambda_{2} \Psi$ in the second.

2. One can obtain alternate spectral resolutions $\left\{\Psi_{j}^{(1)}\right\},\left\{\Psi_{k}^{(2)}\right\}$ for the multiply-degenerate eigenspaces of $H$,

$$
L_{1} \Psi_{j}^{(1)}=\lambda_{1}^{(1)} \Psi_{j}^{(1)}, \quad L_{2} \Psi_{k}^{(2)}=\lambda_{2}^{(2)} \Psi_{k}^{(2)} .
$$

These alternate resolutions resolve the degeneracy problem.

3. The interbasis expansions

$$
\Psi_{k}^{(2)}=\sum_{j} a_{j k} \Psi_{j}^{(1)}
$$

yield important special function identities. In many cases, these become expansions of one set of multivariable orthogonal polynomials in terms of another set.

4. The operators $H, L_{1}, L_{2}$ generate a quadratic algebra. Indeed, with $R=\left[L_{1}, L_{2}\right]$ we have that $R^{2}$ is a polynomial of order 3 in $H, L_{1}, L_{2}$, whereas $\left[L_{1}, R\right]$ and $\left[L_{2}, R\right]$ are polynomials of order 2 in $H, L_{1}, L_{2}$. A corresponding statement is true for algebra generated by the symmetries $\mathcal{H}, \mathcal{L}_{1}, \mathcal{L}_{2}$ under the Poisson bracket. (Note: This is a remarkable 
property, and is false for general symmetries. Consider Euclidean 2space with Hamiltonian $\mathcal{H}=p_{x}^{2}+p_{y}^{2}$. The algebra of all symmetries of $\mathcal{H}$ is generated by $p_{x}, p_{y}, M=x p_{y}-y p_{x}$. Let

$$
\mathcal{L}_{1}=M^{2}+p_{x} p_{y}, \quad \mathcal{L}_{2}=p_{x}^{2} .
$$

Then we have $\mathcal{R}=\left\{\mathcal{L}_{1}, \mathcal{L}_{2}\right\}=4 M p_{x} p_{y}$ and

$$
\mathcal{R}^{2}=\mathcal{F}\left(\mathcal{L}_{0}, \mathcal{L}_{1}, \mathcal{L}_{2}\right)=16 \mathcal{L}_{1} \mathcal{L}_{2}\left(\mathcal{L}_{0}-\mathcal{L}_{2}\right)-16 \mathcal{L}_{2}^{\frac{3}{2}}\left(\mathcal{L}_{0}-\mathcal{L}_{2}\right)^{\frac{3}{2}}
$$

Since $\mathcal{H}, \mathcal{L}_{1}, \mathcal{L}_{2}$ are functionally independent $\mathcal{R}^{2}$ must be a function of these symmetries. However, although $\mathcal{F}$ is defined and bounded at the point $\left(\mathcal{L}_{0}, \mathcal{L}_{1}, \mathcal{L}_{2}\right)=(0,0,0)$, it is not a polynomial, and not even analytic at this point. Thus it has no power series expansion about the origin.)

5. The quadratic algebra structure can be used to compute the interbase expansion coefficients.

\section{$5 \quad$ Examples in higher dimensions}

An extreme case, superintegrability in $n$ dimensions, occurs for the Schrödinger equation

$$
\left(\Delta_{n}+V_{n}(\mathbf{q})\right) \Psi=-M(M+G-1) \Psi
$$

where

$$
\begin{gathered}
V_{n}=-\frac{1}{4} \sum_{i=1}^{n} \frac{\left(\gamma_{i}-\frac{1}{2}\right)\left(\gamma_{i}-\frac{3}{2}\right)}{q_{i}^{2}}-\frac{1}{4} \frac{\left(\gamma_{n+1}-\frac{1}{2}\right)\left(\gamma_{n+1}-\frac{3}{2}\right)}{q_{0}^{2}}+\alpha, \\
\alpha=\frac{1}{4}\left[(1-G)^{2}-1-\frac{(n-3)(n+1)}{4}\right],
\end{gathered}
$$

$G=\sum_{j=1}^{n+1} \gamma_{j}$, and $\Delta_{n}$ is the Laplace-Beltrami operator on the $n$-sphere. Here

$$
q_{0}^{2}+q_{1}^{2}+\cdots+q_{n}^{2}=1 .
$$


One can transfer this Schrödinger equation with a scalar potential $V_{n}$ to one with vector potential $\Lambda_{n}$ through the use of a multiplier transformation $\rho$. Setting $\Phi(\mathbf{x})=\rho(\mathbf{x}) \Psi(\mathbf{x})$ for a nonzero scalar function $\rho$ we find

$$
\begin{aligned}
H \Phi \equiv\left(\Delta_{n}+\Lambda_{n}\right) \Phi & =-M(M+G-1) \Phi \\
\Longleftrightarrow\left(\Delta_{n}+V_{n}(\mathbf{x})\right) \Psi & =-M(M+G-1) \Psi,
\end{aligned}
$$

provided

$$
\rho^{-1}=x_{1}^{\gamma_{1} / 2-1 / 4} \cdots x_{n}^{\gamma_{n} / 2-1 / 4}(1-x)^{\gamma_{n+1} / 2-1 / 4},
$$

and

$$
\Lambda_{n}=\sum_{j=1}^{n}\left[\gamma_{j}-\frac{1}{2}+\left(\frac{n+1}{2}-G\right) x_{j}\right] \partial_{x_{j}} .
$$

Here

$$
H=\sum_{i, j=1}^{n}\left(x_{i} \delta_{i j}-x_{i} x_{j}\right) \partial_{x_{i} x_{j}}+\sum_{i=1}^{n}\left(\gamma_{i}-G x_{i}\right) \partial_{x_{i}}
$$

in the coordinates

$$
\begin{aligned}
q_{0}^{2} & =1-\sum_{i=1}^{n} x_{i}=1-x \\
q_{1}^{2} & =x_{1} \\
q_{2}^{2} & =x_{2} \\
& \vdots \\
q_{n}^{2} & =x_{n} .
\end{aligned}
$$

In the paper [17] and the book [18] all separable coordinates for the equation $\Delta_{n} \Psi=\lambda \Psi$ are constructed, where $\Delta_{n}$ is the Laplace-Beltrami operator on $S^{n}$. It is shown that all separable coordinates are orthogonal and that for each separable coordinate system the corresponding separated solutions are characterized as simultaneous eigenfunctions of a set of $n$ second order commuting symmetry operators for $\Delta_{n}$. Moreover, the equation $\left(\Delta_{n}+V_{n}\right) \Psi=\lambda \Psi$ where the scalar potential takes the form

$$
V_{n}=\sum_{i=1}^{n} \frac{\alpha_{i}}{q_{i}^{2}}+\frac{\alpha_{0}}{q_{0}^{2}}, \quad \alpha_{0}, \alpha_{1}, \ldots, \alpha_{n}
$$


is separable in all the coordinate systems in which the Laplace-Beltrami eigenvalue equation is separable. These results can easily be extended to solutions of

$$
\left(\Delta_{n}+\Lambda_{n}\right) \Phi=\lambda \Phi
$$

through the mappings

$$
\begin{aligned}
\Delta_{n}+\Lambda_{n} & =\rho\left(\Delta_{n}+V_{n}\right) \rho^{-1} \\
S_{i j} & =\rho S_{i j}^{\prime} \rho^{-1} \\
\Phi & =\rho \Psi .
\end{aligned}
$$

Indeed all separable solutions $\Psi$ map to $R$-separable solutions $\Phi$ of (24), [21]. Finally, since $H=\Delta_{n}+\Lambda_{n}$ maps polynomials of maximum order $m_{k}$ in $x_{k}$ to polynomials of the same type, it follows that a basis of separated solutions can be expressed as polynomials in the $x_{i}$. The second-order symmetry operators for this operator can be chosen to be self-adjoint, so the basis of simultaneous eigenfunctions can be chosen to be orthogonal with respect to the inner product

$$
\begin{gathered}
\left(\Phi_{1}, \Phi_{2}\right) \equiv<\Psi_{1}, \Psi_{2}>=\int \cdots \int_{x_{i}>0, x<1} \Phi_{1}(\mathbf{x}) \overline{\Phi_{2}}(\mathbf{x}) \rho^{-2}(\mathbf{x}) d \omega \\
=\int \cdots \int_{x_{i}>0, x<1} \Phi_{1} \overline{\Phi_{2}} d \tilde{\omega} \\
d \tilde{\omega}=x_{1}^{\gamma_{1}-1} \ldots x_{n}^{\gamma_{n}-1}(1-x)^{\gamma_{n+1}-1} d x_{1} \ldots d x_{n} .
\end{gathered}
$$

Thus every separable coordinate system for the Laplace-Beltrami eigenvalue equation on the $n$-sphere yields an orthogonal basis of polynomial solutions of equation (24), hence an orthogonal basis for all $n$-variable polynomials with inner product (26). For details about the bases that can occur and the interbasis expansion coefficients, see [30,31]. Among the special functions that arise are polyspherical harmonics, products of Jacobi polynomials, Heun polynomials, Lame' polynomials, ellipsoidal polynomials, and Lauricella polynomials.

We will look at one more example, the generalized isotropic oscillator in Eucidean 3-space. This is the Schrödinger equation

$$
H \Psi=-\frac{1}{2}\left(\frac{\partial^{2}}{\partial x^{2}}+\frac{\partial^{2}}{\partial y^{2}}+\frac{\partial^{2}}{\partial z^{2}}\right) \Psi+V(x, y, z) \Psi=E \Psi,
$$


with potential

$V(x, y, z)=\frac{\omega^{2}}{2}\left(x^{2}+y^{2}+z^{2}\right)+\frac{1}{2}\left[\frac{\left(k_{1}^{2}-1 / 4\right)}{x^{2}}+\frac{\left(k_{2}^{2}-1 / 4\right)}{y^{2}}+\frac{\left(k_{3}^{2}-1 / 4\right)}{z^{2}}\right]$

where the constant $k_{i} \geq 1 / 2$. For $k_{i}=1 / 2$ we have the ordinary isotropic oscillator potential. The corresponding Schrödinger equation admits solutions via a separation of variables in eight coordinate systems: Cartesian, spherical, sphero-conical, cylindrical polar, cylindrical elliptic, prolate and oblate spheroidal, ellipsoidal. See [32] for the complete details. Here we consider only the quadratic algebra struture.

Note that a basis for the symmetries of Schrödinger's equation with the potential (28) consists of the six operators:

$$
\begin{aligned}
& M_{i}=-D_{i i}-\frac{k_{i}^{2}-\frac{1}{4}}{x_{i}^{2}}, \quad-\mathcal{H}=M_{1}+M_{2}+M_{3} \\
& J_{i j}=L_{i j}^{2}-\left(k_{i}^{2}-\frac{1}{4}\right) \frac{x_{j}^{2}}{x_{i}^{2}}-\left(k_{j}^{2}-\frac{1}{4}\right) \frac{x_{i}^{2}}{x_{j}^{2}}-\frac{1}{2} \quad i, j=1,2,3
\end{aligned}
$$

where $L_{i j}=x_{i} \partial_{x_{j}}-x_{j} \partial_{x_{i}}, D_{i i}=-\partial_{x_{i}}^{2}+\omega^{2} x_{i}^{2}$ - is a diagonal components of the Demkov tensor [33] and we have the notation $x_{1}=x, x_{2}=y, x_{3}=z$.

The commutators of the operators (3)-(4) can be closed to form a quadratic algebra as follows

$\left[M_{i}, M_{j}\right]=0, \quad\left[M_{i}, J_{j k}\right]=0, \quad\left[M_{i}, J_{i j}\right]=Q_{i j}=Q_{[i j]}, \quad\left[J_{i j}, J_{i k}\right]=R_{[i j k]}=R$

where $Q_{i j}$ is totally antisymmetric and the totally antisymmetric quantity $R_{[i j k]}$ is denoted by $R$. Further commutators are calculated to be

$$
\begin{gathered}
{\left[M_{i}, Q_{j k}\right]=0, \quad\left[M_{i}, Q_{i j}\right]=4\left\{M_{i}, M_{j}\right\}+16 J_{i j}, \quad\left[M_{i}, R\right]=4\left\{M_{k}, J_{i j}\right\}-4\left\{M_{j}, J_{i k}\right\}} \\
{\left[J_{i j}, Q_{i j}\right]=4\left\{M_{i}, J_{i j}\right\}-4\left\{M_{j}, J_{i j}\right\}-8\left(k_{j}^{2}-1\right) M_{i}+8\left(k_{i}^{2}-1\right) M_{j},} \\
{\left[J_{i j}, Q_{i k}\right]=4\left\{M_{i}, J_{j k}\right\}-4\left\{M_{j}, J_{i k}\right\}} \\
{\left[J_{i j}, R\right]=4\left\{J_{i j}, J_{j k}\right\}-4\left\{J_{i j}, J_{i k}\right\}-8\left(k_{i}^{2}-1\right) J_{j k}+8\left(k_{j}^{2}-1\right) J_{i k},}
\end{gathered}
$$

where $\{A, B\}=A B+B A$. The expression for the commutators of the $Q$ and $R$ are

$$
\left[Q_{i j}, Q_{i k}\right]=4\left\{M_{i}, Q_{j k}\right\}, \quad\left[Q_{i j}, R\right]=-4\left\{J_{i j}, Q_{i k}\right\}-4\left\{J_{i j}, Q_{j k}\right\}
$$


All the commutators of the operators $M_{i}, J_{m n}, Q_{p q}$ and $R$ can be expressed in terms of quadratic symmetric products of themselves. The algebra therefore is closed quadratically. There are relations between the symmetric products of the generators of this algebra. The exhaustive list of these is as follows.

$$
\begin{gathered}
Q_{i j}^{2}=\frac{8}{3}\left\{J_{i j}, M_{i}, M_{j}\right\}+\frac{64}{3}\left\{M_{i}, M_{j}\right\}+16 \omega^{2} J_{i j}^{2}-16\left(1-k_{j}^{2}\right) M_{i}^{2}-16\left(1-k_{i}^{2}\right) M_{j}^{2} \\
-\frac{128}{3} \omega^{2} J_{i j}-64 \omega^{2}\left(1-k_{i}^{2}\right)\left(1-k_{j}^{2}\right) \\
\left\{Q_{i j}, Q_{i k}\right\}=\frac{8}{3}\left\{J_{i j}, M_{i}, M_{k}\right\}+\frac{8}{3}\left\{J_{i k}, M_{i}, M_{j}\right\}-\frac{8}{3}\left\{J_{j k}, M_{i}, M_{i}\right\} \\
+32 \omega^{2}\left(1-k_{i}^{2}\right)\left\{J_{i j}, J_{i k}\right\}-32\left(1-k_{i}^{2}\right) M_{j} M_{k}-64 \omega^{2}\left(1-k_{i}^{2}\right) J_{j k} \\
\left\{Q_{i j}, R\right\}=\frac{8}{3}\left\{J_{i j}, J_{i j}, M_{k}\right\}-\frac{8}{3}\left\{J_{i j}, J_{i k}, M_{j}\right\}-\frac{8}{3}\left\{J_{i j}, J_{j k}, M_{i}\right\}-\frac{64}{3}\left\{J_{i j}, M_{k}\right\}-\frac{64}{3}\left\{J_{i k}, M_{j}\right\} \\
-\frac{64}{3}\left\{J_{j k}, M_{i}\right\}+16\left(1-k_{i}^{2}\right)\left\{J_{j k}, M_{j}\right\}+16\left(1-k_{j}^{2}\right)\left\{J_{i k}, M_{i}\right\}-64\left(1-k_{i}^{2}\right)\left(1-k_{j}^{2}\right) M_{k} \\
R^{2}=-\frac{4}{3}\left\{J_{i j}, J_{i k}, J_{j k}\right\}+\frac{64}{3}\left\{J_{i j}, J_{i k}\right\}+\frac{64}{3}\left\{J_{i j}, J_{j k}\right\}+\frac{64}{3}\left\{J_{i k}, J_{j k}\right\}-16\left(1-k_{k}^{2}\right) J_{i j}^{2} \\
-16\left(1-k_{j}^{2}\right) J_{i k}^{2}-16\left(1-k_{i}^{2}\right) J_{j k}^{2}+\frac{128}{3}\left(1-k_{k}^{2}\right) J_{i j}+\frac{128}{3}\left(1-k_{j}^{2}\right) J_{i k} \\
+\frac{128}{3}\left(1-k_{i}^{2}\right) J_{j k}+64\left(1-k_{i}^{2}\right)\left(1-k_{j}^{2}\right)\left(1-k_{k}^{2}\right)
\end{gathered}
$$

where $\{A, B, C\}=A B C+C A B+B C A$. Note that only five operators from (3)-(4) are functionally independent [3] and for all the coordinate systems that provide separable solutions for the Schrödinger equation the operators characterizing the separation are always combinations of the $M_{i}$ and $J_{i j}$.

In the limiting case $k_{i}=\frac{1}{2}$, we obtain a quadratic algebra too. In this case

$$
Q_{i j}=2\left(L_{i j} D_{i j}+D_{i j} L_{i j}\right), \quad R=\left\{L_{i k},\left\{L_{i j}, L_{k j}\right\}\right\}
$$

and instead of operators $\left\{M_{i}, J_{i j}, Q_{i j}, R\right\}$ we can consider as a basis for the symmetries the Demkov tensor - $D_{i j}$ and the components of orbital momentum - $L_{i j}$. In this regard we arrive at the Lie algebra corresponding to the symmetries of the isotropic oscillator [33]. 


\section{Some questions and a new approach}

The preceding examples motivate some questions:

1. Is there a uniform procedure to find superintegrable potentials on a constant curvature space?

2. Is there a way of proving completeness, i.e., of demonstrating that all superintegrable potentials for a space have been found?

3. Is superintegrability always associated with multivariable separation?

4. What is the relationship between superintegrability and the existence of quadratic algebras?

First, let us consider the issue of constructing the superintegrable potentials on a given space. We will look at three approaches to the problem.

1. Use what is known from separation of variables theory.

This is the most common approach to finding superintegrable potentials. One classifies the separable systems for the zero potential, determines a general expression for a potential separating in each one of these systems, and then tries to identify potentials that simultaneously separate in several systems. Although the approach is useful for finding examples, there are serious problems in obtaining exhaustive lists. Separable coordinates occur in equivalence classes: two coordinate systems related by a symmetry in the motion group of the space are consided as equivalent. A potential may separate in an ellipsoidal coordinate system, but the ellipsoidal coordinates may not be centered at the origin or aligned with the Cartesian coordinate axes. Such systems are difficult to uncover and many of the lists of superintegrable potentials contain gaps, even for 2-dimensional Minkowski space and hyperboloids. Furthermore, for $n>2$ there is no proof that superintegrability necessarily implies multiseparability.

2. Derive superintegrable potentials on a space as restrictions of free particle systems on higher dimensional spaces. (Split off ignorable coordinates.) 
A very simple example will give the basic idea. Consider the free particle Hamiltonian on Euclidean four-space:

$$
\mathcal{H}_{4}=p_{1}^{2}+p_{2}^{2}+p_{3}^{2}+p_{4}^{4}, \quad d s^{2}=d x_{1}^{2}+d x_{2}^{2}+d x_{3}^{2}+d x_{4}^{2}
$$

There are 4 separable coordinate systems for the Hamilton-Jacobi equation that have two angular variables $\theta_{1}, \theta_{2}$. The simplest of these is

$$
x_{1}=r_{1} \cos \theta_{1}, \quad x_{2}=r_{1} \sin \theta_{1}, \quad x_{3}=r_{2} \cos \theta_{2}, \quad x_{4}=r_{2} \sin \theta_{2} .
$$

The other 3 systems are of the form $u_{1}, u_{2}, \theta_{1}, \theta_{2}$ where $r_{j}=f_{j}\left(u_{1}, u_{2}\right), j=$ 1,2 . In the new coordinates we have

$$
\mathcal{H}_{4}=p_{r_{1}}^{2}+p_{r_{2}}^{2}+\frac{p_{\theta_{1}}^{2}}{r_{1}^{2}}+\frac{p_{\theta_{2}}^{2}}{r_{2}^{2}}, \quad d s^{2}=d r_{1}^{2}+d r_{2}^{2}+r_{1}^{2} d \theta_{1}^{2}+r_{2}^{2} d \theta_{2}^{2}
$$

Note that the $\theta_{j}$ are ignorable coordinates, i.e., the metric tensor in the new coordinates is independent of the $\theta_{j}$. Now restrict to the symplectic submanifold such that $p_{\theta_{j}}=\alpha_{j}, \quad d \theta_{j}=0$ where the $\alpha_{j}$ are constants. Then the Hamiltonian becomes

$$
\mathcal{H}_{4}=p_{r_{1}}^{2}+p_{r_{2}}^{2}+\frac{\alpha_{1}^{2}}{r_{1}^{2}}+\frac{\alpha_{2}^{2}}{r_{2}^{2}}, \quad d s^{2}=d r_{1}^{2}+d r_{2}^{2}
$$

Locally, we can regard the Hamiltonian as defined in Euclidean 2-space and with the potential $V=\frac{\alpha_{1}^{2}}{r_{1}^{2}}+\frac{\alpha_{2}^{2}}{r_{2}^{2}}$. The restricted Hamiltonian-Jacobi equation remains separable in 4 cordinate systems $\left\{u_{1}, u_{2}\right\}$, hence this is a superintegrable system. Boyer, Kalnins and Winternitz have applied this idea to the restriction of a free Hamiltonian on Hermitian hyperbolic spaces to hyperboloids in order to derive previously unknown superintegrable systems, $[34,35]$. This is a powerful method, but there is no evident way to prove by this approach that all superintegrable systems have been obtained.

3. New way: use integrabilty conditions. This last approach is the one that we shall advocate in the remainder of this paper. 


\section{Complex Euclidean 2-space treated in de- tail}

We consider, in detail, the case

$$
\mathcal{H}=p_{1}^{2}+p_{2}^{2}+V(x, y)
$$

where $p_{1}=p_{x}, p_{2}=p_{y}$ and all variables are complex. Let us assume that in

addition to the classical Hamiltonian we have two quadratic constants of the motion

$$
\mathcal{L}_{h}=\sum_{k, j=1}^{2} a_{(h)}^{k j}(x, y) p_{k} p_{j}+W_{(h)}(x, y) \equiv \ell_{h}+W_{(h)}, \quad h=1,2
$$

which must satisfy

$$
\left\{\mathcal{H}, \mathcal{L}_{h}\right\}=0
$$

with \{\} the usual Poisson bracket. We require that the set $\left\{d \mathcal{H}, d \mathcal{L}_{1}, d \mathcal{L}_{2}\right\}$ is linearly independent, so that $\mathcal{H}, \mathcal{L}_{1}, \mathcal{L}_{2}$ is a maximal set of functionally independent constants of the motion. It is clear that $\mathcal{R}=\left\{\mathcal{L}_{1}, \mathcal{L}_{2}\right\}$ is a constant of the motion, so it and $\mathcal{R}^{2}$ must be expressible as an analytic function of $\mathcal{H}, \mathcal{L}_{1}, \mathcal{L}_{2}$ :

$$
\mathcal{R}^{2}=\mathcal{F}\left(\mathcal{L}_{0}, \mathcal{L}_{1}, \mathcal{L}_{2}\right), \quad \mathcal{H} \equiv \mathcal{L}_{0}
$$

Note that $\mathcal{R}$ has the form

$$
\mathcal{R}=\sum_{k, l, m=1}^{2} c^{k l m} p_{k} p_{l} p_{m}+\sum_{k=1}^{2} d^{k} p_{k}
$$

but that it doesn't follow that $\mathcal{R}^{2}$ is necessarily a polynomial as a function of $\mathcal{L}_{0}, \mathcal{L}_{1}, \mathcal{L}_{2}$. We will find conditions that guarantee that $\mathcal{F}$ is a third-order polynomial in its arguments.

Using the identity

$$
\{\mathcal{K}, \mathcal{G}\}=\sum_{h=0}^{2}\left\{\mathcal{K}, \mathcal{L}_{h}\right\} \frac{\partial \mathcal{G}}{\partial \mathcal{L}_{h}}
$$

for a continuously differentiable function $\mathcal{G}\left(\mathcal{L}_{h}\right)$, we find the relations

$$
\left\{\mathcal{L}_{1}, \mathcal{R}\right\}=\frac{1}{2} \frac{\partial \mathcal{F}}{\partial \mathcal{L}_{2}}, \quad\left\{\mathcal{L}_{2}, \mathcal{R}\right\}=-\frac{1}{2} \frac{\partial \mathcal{F}}{\partial \mathcal{L}_{1}}
$$


Thus, the constants of the motion $\left\{\mathcal{L}_{1}, \mathcal{R}\right\},\left\{\mathcal{L}_{2}, \mathcal{R}\right\}$ are easily computed once $\mathcal{F}$ is known. Further, if $\mathrm{F}$ is a polynomial in the invariants, then so are $\left\{\mathcal{L}_{1}, \mathcal{R}\right\}$, and $\left\{\mathcal{L}_{2}, \mathcal{R}\right\}$.

We first determine the conditions that the function

$$
\mathcal{L}=\sum_{j, k=1}^{2} a^{j k}(x, y) p_{k} p_{j}+W(x, y), \quad a^{j k}=a^{k j}
$$

must satisfy to be a constant of the motion. The requirement is $\{\mathcal{H}, \mathcal{L}\}=0$ where

$$
\{f, g\}=\sum_{j=1}^{2}\left(\frac{\partial f}{\partial p_{j}} \frac{\partial g}{\partial x_{j}}-\frac{\partial f}{\partial x_{j}} \frac{\partial g}{\partial p_{j}}\right), \quad\left(x_{1}, x_{2}\right)=(x, y),
$$

and

$$
\mathcal{H}=p_{1}^{2}+p_{2}^{2}+V(x, y) .
$$

The conditions are thus

$$
\begin{array}{ll}
\frac{\partial a^{11}}{\partial x}=0 & 2 \frac{\partial a^{12}}{\partial x}+\frac{\partial a^{11}}{\partial y}=0 \\
\frac{\partial a^{22}}{\partial y}=0 & \frac{\partial a^{22}}{\partial x}+2 \frac{\partial a^{12}}{\partial y}=0
\end{array}
$$

and

$$
\frac{\partial W}{\partial x}-a^{11} \frac{\partial V}{\partial x}-a^{12} \frac{\partial V}{\partial y}=0, \quad \frac{\partial W}{\partial y}-a^{12} \frac{\partial V}{\partial x}-a^{22} \frac{\partial V}{\partial y}=0 .
$$

The solution for the terms quadratic in the $p_{j}$ is

$$
\begin{aligned}
a^{11} & =\alpha_{1} y^{2}+\alpha_{2} y+\alpha_{3}^{\prime} \\
a^{12} & =-\alpha_{1} x y-\frac{1}{2} \alpha_{2} x-\frac{1}{2} \alpha_{4} y+\frac{1}{2} \alpha_{5} \\
a^{22} & =\alpha_{1} x^{2}+\alpha_{4} x+\alpha_{3}^{\prime \prime},
\end{aligned}
$$

where the $\alpha_{k}$ are constants. The requirement that $\partial_{x} W_{y}=\partial_{y} W_{x}$ leads from (41) to the second order partial differential equation for the potential

$$
\begin{aligned}
& \frac{1}{2}\left(2 \alpha_{1} x y+\alpha_{2} x+\alpha_{4} y-\alpha_{5}\right)\left(V_{x x}-V_{y y}\right)+\left(\alpha_{1}\left[y^{2}-x^{2}\right]+\alpha_{2} y-\alpha_{4} x+\alpha_{3}\right) V_{x y} \\
& =\left(-3 \alpha_{1} y-\frac{3}{2} \alpha_{2}\right) V_{x}+\left(3 \alpha_{1} x+\frac{3}{2} \alpha_{4}\right) V_{y},
\end{aligned}
$$


where $\alpha_{3}=\alpha_{3}^{\prime}-\alpha_{3}^{\prime \prime}$. This is a fundamental equation in our approach. We denote the solution space of this equation by

$$
\left[\alpha_{1}, \cdots, \alpha_{5}\right]
$$

Let us now return to our assumption that the Hamilton-Jacobi equation admits two constants of the motion:

$$
\mathcal{L}_{h}=\sum_{j, k=1}^{2} a_{(h)}^{j k} p_{k} p_{j}+W_{(h)}, \quad h=1,2 .
$$

These two operators together with $\mathcal{H}$ are assumed functionally independent. The constant of the motion $\mathcal{L}_{1}$ leads to the condition (46) on the potential $V$; whereas $\mathcal{L}_{2}$ leads to the second condition

$$
\left[\beta_{1}, \cdots, \beta_{5}\right]
$$

The potential must lie in the intersection of the solution spaces $(46,47)$ for these two conditions. It follows that the equations

$$
V_{x x}-V_{y y}=A V_{x}+B V_{y}, \quad V_{x y}=C V_{x}+D V_{y}
$$

must hold, where

$$
\begin{aligned}
A \mathcal{E} & =\frac{3}{2} H_{12}\left(x^{2}+y^{2}\right)-3 H_{14} x y+3 H_{13} y-\frac{3}{2} H_{24} x+\frac{3}{2} H_{23} \\
B \mathcal{E} & =\frac{3}{2} H_{14}\left(x^{2}+y^{2}\right)-3 H_{12} x y-3 H_{13} x+\frac{3}{2} H_{24} y+\frac{3}{2} H_{34} \\
2 C \mathcal{E} & =-3 H_{14} y^{2}+\left(-\frac{3}{2} H_{24}+3 H_{15}\right) y+\frac{3}{2} H_{25} \\
2 D \mathcal{E} & =3 H_{12} x^{2}+\left(-\frac{3}{2} H_{24}-3 H_{15}\right) x-\frac{3}{2} H_{45} \\
2 \mathcal{E} & =-H_{12} x y^{2}+H_{14} x^{2} y-H_{12} x^{3}+H_{14} y^{3}-2 H_{13} x y+H_{24}\left(x^{2}+y^{2}\right) \\
& +H_{15}\left(x^{2}-y^{2}\right)+\left(H_{34}-H_{25}\right) y+\left(H_{45}-H_{23}\right) x-H_{35},
\end{aligned}
$$

and $H_{k \ell}=-H_{\ell k}=\alpha_{k} \beta_{\ell}-\alpha_{\ell} \beta_{k}$.

From the fundamental equations (48) we can compute all of the third partial derivatives of $V$. Indeed

$$
V_{x x x}=\left(A_{x}+B C+C_{y}+C^{2}+A^{2}\right) V_{x}+\left(B_{x}+D B+D_{y}+C D+A B\right) V_{y}
$$




$$
\begin{aligned}
& +(A+D) V_{y y} \\
V_{x x y} & =\left(C_{x}+D C+A C\right) V_{x}+\left(D_{x}+D^{2}+B C\right) V_{y}+C V_{y y} \\
V_{x y y} & =\left(C_{y}+C^{2}\right) V_{x}+\left(D_{y}+C D\right) V_{y}+D V_{y y} \\
V_{y y y} & =\left(-A_{y}+C_{x}+D C\right) V_{x}+\left(-B_{y}-A D+D_{x}+D^{2}+B C\right) V_{y} \\
& +(C-B) V_{y y} .
\end{aligned}
$$

Thus if the potential $V$ is subject to the two conditions $(46,47)$, then $V$ can depend on at most 3 parameters, in addition to a trivial additive constant. We can choose these parameters to be $V_{x}\left(x_{0}, y_{0}\right), V_{y}\left(x_{0}, y_{0}\right), V_{y y}\left(x_{0}, y_{0}\right)$ for any fixed regular point $\left(x_{0}, y_{0}\right)$. Then $V_{x x}\left(x_{0}, y_{0}\right)$ and all higher derivatives can be computed by successive differentiation of relations (50). We require that our potential be nondegenerate, i.e., that it depend on 3 arbitrary parameters.

Then, the conditions $\partial_{x} V_{x x y}=\partial_{y} V_{x x x}, \partial_{y} V_{x x y}=\partial_{x} V_{x y y}, \partial_{y} V_{x y y}=\partial_{x} V_{y y y}$ for the fourth partial derivatives lead to the integrability conditions

$$
\begin{aligned}
\partial_{x}(2 C-B) & =\partial_{y}(2 D+A) \quad \text { (satisfied identically) } \\
C_{x x}-C_{y y}-A_{x y} & =2 C C_{y}-D A_{y}-2 C D_{x}+A A_{y}-A C_{x} \\
& +C B_{y}+B C_{y} \\
D_{x x}-D_{y y}-B_{x y} & =-2 D D_{x}-C B_{x}+2 D C_{y}-B B_{x} \\
& -B D_{y}+D A_{x}+A D_{x} .
\end{aligned}
$$

Note that if we have another constant of the motion $\mathcal{L}_{3}$ associated with a nondegenerate potential, then $\mathcal{L}_{3}$ must be a linear combination of $\mathcal{H}, \mathcal{L}_{1}, \mathcal{L}_{2}$. Indeed, if $\mathcal{L}_{3}$ is not a linear combination of the basis functions, then the potential $V$ must satisfy an equation (45) that is linearly independent of the equations associated with $\mathcal{L}_{1}, \mathcal{L}_{2}$. This means an additional constraint on the solution space and that $V$ can depend on at most two parameters, which is a contradiction.

We will use the conditions $(52,53)$ to classify the possible potentials $V$ and the corresponding constants of the motion $\mathcal{L}_{1}, \mathcal{L}_{2}$. For this we note that it is only the three-dimensional subspace spanned by $\mathcal{H}, \mathcal{L}_{1}, \mathcal{L}_{2}$ that matters; we can choose any basis for this subspace. Hence we can replace the conditions $(46,47)$ by linear combinations of themselves without changing the potential. Moreover, to simplify the results we note that we can always subject the coordinates $(x, y)$, and $\mathcal{L}_{1}, \mathcal{L}_{2}$ to a simultaneous Euclidean motion, i.e., we regard all translated and rotated potentials as members of the same equivalence class. 
Multiplying both sides of (52) and (53) by $\mathcal{E}^{3}$ we obtain polynomial identities in $x$ and $y$. Equating the coefficients of the various powers $x^{n} y^{m}$ we obtain conditions on the parameters $H_{j k}$. The simplest non-trivial condition, which is associated with the coefficient of a fifth order power in either of the equations, is

$$
2 H_{15}\left(H_{14}^{2}-H_{12}^{2}\right)+H_{24}\left(H_{14}^{2}+H_{12}^{2}\right)-4 H_{14} H_{12} H_{13}=0 .
$$

We exploit these and the remaining conditions, and Euclidean motions to classify the possibilities for the $\mathcal{L}_{j}$. The full conditions (52) and (53), expressed in terms of the parameter $H_{i j}$, take several pages to list and are complicated to solve directly. (Indeed a symbol manipulation program was an important aid to our computations.) However, by dividing the problem up into special cases and using Euclidean motions, we can simplify the conditions and obtain a full solution. In the listing that follows we use the fact that the constants of the motion can each be expressed as a quadratic element in the enveloping algebra of the Euclidean group in the plane with basis elements

$$
p_{x}, p_{y}, M=x p_{y}-y p_{x},
$$

to which a potential term $W(x, y)$ is added. (Strictly speaking, conditions (52) and (53) are only necessary conditions for existence of nondegenerate potentials. However, in our case-by-case study we have found that they are also sufficient: all solutions of these equations lead to nondegenerate potentials.)

Suppose $I \equiv H_{12}^{2}+H_{14}^{2} \neq 0$. Via an appropriate coordinate rotation through complex angle $\theta$ we obtain a new set of equations (48) in the rotated coordinates where the new parameters $H_{12}^{\prime}, H_{14}^{\prime}$ are related to the original ones by

$$
H_{12}^{\prime}=H_{12} \cos \theta+H_{14} \sin \theta, \quad H_{14}^{\prime}=H_{14} \cos \theta-H_{12} \sin \theta,
$$

and for which $I=I^{\prime}$. Thus, by an appropriate choice of $\theta$, we can assume $H_{12}=0$. Then, by an appropriate Euclidean translation that leaves $H_{12}, H_{14}$ unchanged, it follows from (49) that we can assume $H_{13}=H_{24}=0$. Then (54) implies $H_{15}=0$, so $H_{35}=H_{23}=0$. Further, the fourth order integrability conditions give $\mathrm{H}_{34} \mathrm{H}_{45}=0$, and under appropriate translations, we can take $H_{34}=H_{45}=0$. 
Case (1) $\quad H_{12}^{2}+H_{14}^{2} \neq 0$

$$
[1,0,0,0,0], \quad[0,0,0,1,0]
$$

Here,

$$
\begin{aligned}
L_{1} & =4 M^{2}+W^{(1)}, \quad L_{2}=-2 M p_{y}+W^{(2)} \\
V(x) & =\frac{\alpha}{\sqrt{x^{2}+y^{2}}}+\frac{1}{\sqrt{x^{2}+y^{2}}}\left[\frac{\beta}{\sqrt{x^{2}+y^{2}}+x}+\frac{\gamma}{\sqrt{x^{2}+y^{2}}-x}\right] .
\end{aligned}
$$

This potential allows separation in parabolic or polar coordinates.

We obtain the remaining cases by continuing in this way; for details see [36]. The results are:

\section{Case (2a)}

$$
\left[1,0,2 c^{2}, 0,0\right], \quad[0,-1, i c, i,-c]
$$

where,

$$
\begin{aligned}
L_{1} & =M^{2}+c^{2} p_{x}^{2}+W^{(1)}, \quad L_{2}=M p_{+}+\frac{i c}{2} p_{+}^{2}+W^{(2)} \\
V(x) & =\frac{\alpha z}{\left(c^{2}-z^{2}\right)^{\frac{1}{2}}}+\frac{\beta}{\sqrt{(c-z)(c+\bar{z})}}+\frac{\gamma}{\sqrt{(c+z)(c+\bar{z})}} .
\end{aligned}
$$

The corresponding Hamilton-Jacobi and Schrödinger equations for this system separates in elliptical coordinates, as well as shifted elliptical coordinates. Case (2b)

$$
[1,0,2,0,2 i], \quad[0,-1,2 i, i,-2]
$$

where,

$$
\begin{aligned}
L_{1} & =M^{2}+p_{+}^{2}+W^{(1)}, \quad L_{2}=\left(M+2 i p_{+}\right)^{2}+p_{+}^{2}+W^{(2)} \\
V(x) & =\frac{\alpha}{z^{2}}+\frac{\beta}{\sqrt{z^{3}(\bar{z}+2)}}+\frac{\gamma}{\sqrt{z(\bar{z}+2)}} .
\end{aligned}
$$

This system separates in terms of hyperbolic coordinates and displaced hyperbolic coordinates. 
Case (3a) $\quad H_{15}^{2}+H_{13}^{2} \neq 0$

$$
[1,0,0,0,0], \quad[0,0,1,0,0]
$$

Here,

$$
\begin{aligned}
L_{1} & =M^{2}+W^{(1)}, \quad L_{2}=p_{x}^{2}+W^{(2)} \\
V(x) & =\alpha\left(x^{2}+y^{2}\right)+\frac{\beta}{x^{2}}+\frac{\gamma}{y^{2}} .
\end{aligned}
$$

This potential permits separation in polar, elliptic and cartesian coordinates.

Case (3b) $H_{15}^{2}+H_{13}^{2}=0$

$$
[1,0,0,0,0], \quad[0,0,2,0, \pm 2 i]
$$

Here,

$$
\begin{aligned}
L_{1} & =M^{2}+W^{(1)}, \quad L_{2}=p_{+}^{2}+W^{(2)} \\
V(x) & =\alpha \frac{x^{2}+y^{2}}{(x+i y)^{4}}+\frac{\beta}{(x+i y)^{2}}+\gamma\left(x^{2}+y^{2}\right) .
\end{aligned}
$$

(There is a similar solution where the term $p_{+}^{2}$ in $L_{2}$ is replaced by $p_{-}^{2}$.) The potential permits separation in hyperbolic and polar coordinates.

Case (3c) $\quad H_{15}^{2}+H_{13}^{2}=0$

$$
\left[1,0, c^{2}, 0,0\right], \quad[0,0,2,0, \pm 2 i]
$$

Here,

$$
\begin{aligned}
L_{1} & =M^{2}+c^{2} p_{x}^{2}+W^{(1)}, \quad L_{2}=p_{+}^{2}+W^{(2)} \\
V(x) & =\frac{\alpha z}{\sqrt{z^{2}-c^{2}}}+\frac{\beta \bar{z}}{\sqrt{z^{2}-c^{2}}\left(z+\sqrt{z^{2}-c^{2}}\right)^{2}}+\gamma z \bar{z}
\end{aligned}
$$

The potential permits separation in hyperbolic and elliptic coordinates.

Case (4) $H_{12}=H_{13}=H_{14}=H_{15}=0, \alpha_{2} \neq 0, H_{24} \neq 0$

$$
[0,1,0,0,0], \quad[0,0,0,1,0]
$$


Here,

$$
\begin{aligned}
L_{1} & =-2 M p_{x}+W^{(1)}, \quad L_{2}=-2 M p_{y}+W^{(2)} \\
V(x) & =\frac{\alpha}{\sqrt{x^{2}+y^{2}}}+\beta \frac{\left(\sqrt{x^{2}+y^{2}}+x\right)^{\frac{1}{2}}}{\sqrt{x^{2}+y^{2}}}+\gamma \frac{\left(\sqrt{x^{2}+y^{2}}-x\right)^{\frac{1}{2}}}{\sqrt{x^{2}+y^{2}}} .
\end{aligned}
$$

Separation of variables is possible in two types of parabolic coordinates, the usual parabolic coordinates and the interchanged parabolic coordinates $x=$ $\mu \nu, y=\frac{1}{2}\left(\mu^{2}-\nu^{2}\right)$.

Case (5) $\quad\left(H_{34}-H_{25}\right)^{2}+\left(H_{45}-H_{23}\right)^{2} \neq 0$

$$
\left[0,1, \alpha_{3}, \pm i, 0\right], \quad[0,0, \pm i, 0,1]
$$

Here we choose the typical case

$$
\begin{aligned}
L_{1} & =4 i M p_{-}+p_{+}^{2}+W^{(1)}, \quad L_{2}=p_{-}^{2}+W^{(2)} \\
V(x) & =\alpha(x-i y)+\beta\left(x+i y-\frac{3}{2}(x-i y)^{2}\right)+\gamma\left(x^{2}+y^{2}-\frac{1}{2}(x-i y)^{3}\right) .
\end{aligned}
$$

The possible separable coordinates are semihyperbolic coordinates corresponding to operator $M p_{-}+p_{+}^{2}$ and shifted semihyperbolic coordinates with operator $M p_{-}+\delta p_{-}^{2}+p_{+}^{2}$. This corresponds to the standard coordinates shifted via the transformation $x \rightarrow x+\delta, y \rightarrow y+i \delta$.

Case (6a) $\quad H_{45}=0$

$$
[0,0,1,0,0], \quad[0,0,0,1,0]
$$

Here,

$$
\begin{aligned}
L_{1} & =p_{x}^{2}+W^{(1)}, \quad L_{2}=-2 M p_{y}+W^{(2)} \\
V(x) & =\alpha\left(4 x^{2}+y^{2}\right)+\beta x+\frac{\gamma}{y^{2}} .
\end{aligned}
$$

The possible separable coordinates are cartesian and parabolic.

Case (6b) $\quad H_{45} \neq 0$

$$
[0,0,1,0, \pm i], \quad[0,0,0,1,0]
$$


Here we take

$$
\begin{aligned}
L_{1} & =2 p_{y} p_{+}+W^{(1)}, \quad L_{2}=M p_{y}+W^{(2)} \\
V(x) & =\frac{\alpha}{\sqrt{x+i y}}+\beta x+\gamma \frac{2 x+i y}{\sqrt{x+i y}} .
\end{aligned}
$$

There is the possibility of separability in parabolic coordinates $\left\{M p_{y}\right\}$ or displaced parabolic coordinates $\left\{\left(M+\delta\left(p_{x} \pm i p_{y}\right)\right) p_{y}\right\}$ for suitable $\delta$.

This is the complete list of superintegrable systems in complex Euclidean 2-space. It includes real Euclean space and Minkowski space as special cases. Now we demonstrate that there is a quadratic algebra associated with each nondegenerate potential. Because we are working in two dimensions there can only be three functionally independent constants at most. Consequently all Poisson brackets must be functionally dependent on $\mathcal{H}=\mathcal{L}_{0}, \mathcal{L}_{1}$ and $\mathcal{L}_{2}$. We want to show that in fact $\mathcal{R}^{2}=\left\{\mathcal{L}_{1}, \mathcal{L}_{2}\right\}^{2}=\mathcal{F}\left(\mathcal{L}_{0}, \mathcal{L}_{1}, \mathcal{L}_{2}\right)$ is a polynomial in these variables.

Note that for arbitrary $\mathcal{L}_{1}, \mathcal{L}_{2}$, the $\mathcal{F}$ is in general not a polynomial. Consider the example:

$$
\mathcal{L}_{0}=p_{x}^{2}+p_{y}^{2}, \quad \mathcal{L}_{1}=M^{2}+p_{x} p_{y}, \quad \mathcal{L}_{2}=p_{x}^{2} .
$$

Then we have $\mathcal{R}=\left\{\mathcal{L}_{1}, \mathcal{L}_{2}\right\}=4 M p_{x} p_{y}$ and

$$
\mathcal{R}^{2}=\mathcal{F}\left(\mathcal{L}_{0}, \mathcal{L}_{1}, \mathcal{L}_{2}\right)=16 \mathcal{L}_{1} \mathcal{L}_{2}\left(\mathcal{L}_{0}-\mathcal{L}_{2}\right)-16 \mathcal{L}_{2}^{\frac{3}{2}}\left(\mathcal{L}_{0}-\mathcal{L}_{2}\right)^{\frac{3}{2}}
$$

Here, although $\mathcal{F}$ is defined and bounded at the point $\left(\mathcal{L}_{0}, \mathcal{L}_{1}, \mathcal{L}_{2}\right)=(0,0,0)$, it is not analytic at this point. Thus it has no power series expansion about the origin. We conjecture that this is an illustration of the general problem: if $\mathcal{F}$ is not a polynomial, then there are branch points or cuts at $(0,0,0)$.

We will show, however, that for nondegenerate potentials the associated $\mathcal{F}$ is a polynomial. First, we can verify that this is true when the potential is turned off, i.e., if we consider only the functions

$$
\ell_{h}=\sum_{j, k=1}^{2} a_{(h)}^{j k} p_{k} p_{j}, i=h, 2 \quad \ell_{0}=p_{x}^{2}+p_{y}^{2}
$$


where $\mathcal{L}_{h}=\ell_{h}+W^{(h)}$. Let $\mathcal{R}=\left\{\ell_{1}, \ell_{2}\right\}$. Then for each of the cases listed above it is straightforward to check that $\mathcal{R}^{2}=\mathcal{P}_{3}\left(\ell_{0}, \ell_{1}, \ell_{2}\right)$ where $\mathcal{P}_{3}$ is a homogeneous third order polynomial in its arguments. ${ }^{1}$ It follows that

$$
\mathcal{R}^{2}=\mathcal{F}\left(\mathcal{L}_{0}, \mathcal{L}_{1}, \mathcal{L}_{2}\right)=\mathcal{P}_{3}\left(\mathcal{L}_{0}, \mathcal{L}_{1}, \mathcal{L}_{2}\right)+\mathcal{F}_{4}\left(\mathbf{s}, \mathcal{L}_{0}, \mathcal{L}_{1}, \mathcal{L}_{2}\right)
$$

where $\mathcal{F}_{4}$ is a fourth, second and zeroth order polynomial in the momenta $p_{x}, p_{y}$, and $\mathcal{F}_{4}\left(\mathbf{0}, \mathcal{L}_{0}, \mathcal{L}_{1}, \mathcal{L}_{2}\right)=0$. Here, the parameters in the potential are denoted by $\mathbf{s}=\left(V_{x}^{0}, V_{y}^{0}, V_{y y}^{0}\right)$, evaluated at some fixed point $\left(x_{0}, y_{0}\right)$ and $\mathcal{F}_{4}$ is a polynomial function of these parameters.

From (36) we have

$$
\begin{gathered}
\left\{\ell_{1}, \mathcal{R}\right\}=\frac{1}{2} \frac{\partial \mathcal{P}_{3}}{\partial \ell_{2}}\left(\ell_{0}, \ell_{1}, \ell_{2}\right) \\
\left\{\ell_{2}, \mathcal{R}\right\}=-\frac{1}{2} \frac{\partial \mathcal{P}_{3}}{\partial \ell_{1}}\left(\ell_{0}, \ell_{1}, \ell_{2}\right)
\end{gathered}
$$

hence

$$
\begin{gathered}
\left\{\mathcal{L}_{1}, \mathcal{R}\right\}=\frac{1}{2} \frac{\partial \mathcal{P}_{3}}{\partial \mathcal{L}_{2}}\left(\mathcal{L}_{0}, \mathcal{L}_{1}, \mathcal{L}_{2}\right)+\frac{1}{2} \frac{\partial \mathcal{F}_{4}}{\partial \mathcal{L}_{2}}(\mathbf{s}) \\
\left\{\mathcal{L}_{2}, \mathcal{R}\right\}=-\frac{1}{2} \frac{\partial \mathcal{P}_{3}}{\partial \mathcal{L}_{1}}\left(\mathcal{L}_{0}, \mathcal{L}_{1}, \mathcal{L}_{2}\right)-\frac{1}{2} \frac{\partial \mathcal{F}_{4}}{\partial \mathcal{L}_{1}}(\mathbf{s})
\end{gathered}
$$

where the $\partial \mathcal{F}_{4} / \partial \mathcal{L}_{h}(\mathbf{s})$ have only terms of orders two and zero in the momenta. It follows that the $\partial \mathcal{F}_{4} / \partial \mathcal{L}_{h}(\mathbf{s})$ must be expressible as linear combinations of the $\mathcal{L}_{h}$. This shows that the commutators $\left\{\mathcal{L}_{h}, \mathcal{R}\right\}$ can be expressed as polynomials in $\mathcal{L}_{0}, \mathcal{L}_{1}, \mathcal{L}_{2}$. It is then a simple matter to verify that $\mathcal{F}$ itself is a polynomial in $\mathcal{L}_{0}, \mathcal{L}_{1}, \mathcal{L}_{2}$.

A complete list of the quadratic algebra relations for each of the cases studied above is given in [36]. In view of relations (36) it is sufficient to give the relation $R^{2}=F\left(L_{0}, L_{1}, L_{2}\right)$ for each case. An example is

Case (1) $\quad[1,0,0,0,0], \quad[0,0,0,1,0]$

$\mathcal{R}^{2}=16 \mathcal{L}_{1}^{2} \mathcal{H}-16 \mathcal{L}_{2}^{2} \mathcal{L}_{1}-32(\beta+\gamma) \mathcal{L}_{2}^{2}+64 \alpha(\beta-\gamma) \mathcal{L}_{2}+16 \alpha^{2} \mathcal{L}_{1}-256 \beta \gamma \mathcal{H}-32 \alpha^{2}(\beta+\gamma)$

\footnotetext{
${ }^{1}$ Moreover, it is straightforward to verify that the cases corresponding to nondegenerate potentials are the only cases where $\mathcal{P}_{3}$ is a homogeneous third order polynomial in its arguments. Thus the possible quadratic algebras generated by second order elements in the Euclidean Lie algebra correspond one-to-one with nondegenerate potentials.
} 
There are analogous quantum algebras for superintegrable systems arising from the potentials we have already computed. The only difference is that the Poisson bracket is now replaced by the commutator bracket $[A, B]=$ $A B-B A$ and the operators $H, L_{1}$ and $L_{2}$ are the obvious (formally selfadjoint) symmetry partial differential operators.

$$
H=\partial_{x}^{2}+\partial_{y}^{2}+V(x, y), \quad L_{h}=\sum_{k, j=1}^{2} \partial_{k}\left(a_{(h)}^{k j}\right) \partial_{j}+W_{(h)}(x, y), \quad h=1,2 .
$$

Just as for the Hamilton-Jacobi case, if we have another constant of the motion $L$ associated with a maximal potential, then $L$ must be a linear combination of $H, L_{1}, L_{2}$. Indeed, if $L$ is in self-adjoint form, then the conditions that $[H, L]=0$ are identical with (40), (41). Thus, if $L$ is not a linear combination of the basis functions, then the potential $V$ must satisfy an equation (45) that is linearly independent of the equations associated with $L_{1}, L_{2}$. This means an additional constraint on the solution space and that $V$ can depend on at most two parameters, which is a contradiction.

Furthermore the proof of the existence of quadratic algebra relations goes through almost unchanged for the operator case: $\left[L_{1}, L_{2}\right]^{2}=R^{2}$ and $\left[L_{1}, R\right],\left[L_{2}, R\right]$ can be expressed as (symmetric) polynomials in the operators $H, L_{1}, L_{2}$. To make the prior construction go through, one need only note that since $R^{2}$ is a formally self-adjoint 6th order differential symmetry operator, the 5th order terms are fixed linear functions of the 6th order terms. The expressions $\{A, B\}=A B+B A$ and $\{A, B, C\}=A B C+C A B+B C A$ are operator symmetrizers. The detailed results are contained in [36]. An example is

Case (1)

$$
\begin{gathered}
{\left[L_{2}, R\right]=8 L_{2}^{2}+8 H L_{2}+8 \alpha^{2},} \\
{\left[L_{1}, R\right]=8\left\{L_{2}, L_{1}\right\}+16(1+2 \beta+2 \gamma) L_{2}+32 \alpha(\gamma-\beta),} \\
R^{2}=16 L_{1}^{2} H-\frac{8}{3}\left\{L_{2}, L_{2}, L_{1}\right\}-16\left(2 \beta+2 \gamma+\frac{11}{3}\right) L_{2}^{2}-\frac{176}{3} H L_{1} \\
+64 \alpha(\beta-\gamma) L_{2}+16 \alpha^{2} L_{1}+\left(-\frac{32}{3}+96 \gamma+96 \beta+256 \beta \gamma\right) H \\
-\frac{32}{3} \alpha^{2}(3 \beta+3 \gamma-1) .
\end{gathered}
$$




\section{Conclusions}

We have used the concept of a "nondegenerate potential" to add structure to the study of superintegrable classical and quantum mechanical systems in $E_{2, C}$. We have shown how to classify all such systems in a straightforward manner. Furthermore:

1. Each system is associated with a pair of constants of the motion in the classical case, and a pair of symmetry operators in the quantum case, that generate a quadratic algebra.

2. There is a one-to-one correspondence between superintegrable systems and free-field symmetry operators that generate quadratic algebras.

3. Superintegrability implies multiseparability.

We have analogous results for superintegrable systems on the complex 2 -sphere, [37]. The real sphere and the real hyperboloid are special cases. Again we find and fill gaps in the known list of superintegrable systems. The next major challenge is to extend this analysis to higher dimensional systems.

\section{References}

[1] N.W.Evans. Superintegrability in Classical Mechanics; Phys.Rev. A 41 (1990) 5666; Group Theory of the Smorodinsky-Winternitz System; J.Math.Phys. 32, 3369 (1991).

[2] N.W.Evans. Superintegrability of the Calogero-Moser System. Phys. Lett. A 95, 279 (1983).

[3] N.W.Evans. Super-Integrability of the Winternitz System; Phys.Lett. A 147, 483-486, (1990).

[4] L.P.Eisenhart. Enumeration of Potentials for Which One-Particle Schrödinger Equations Are Separable; Phys.Rev. 74, 87 (1948).

[5] J.Friš, V.Mandrosov, Ya.A.Smorodinsky, M.Uhlir and P.Winternitz. On Higher Symmetries in Quantum Mechanics; Phys.Lett. 16, 354 (1965). 
[6] J.Friš, Ya.A.Smorodinskii, M.Uhlír and P.Winternitz. Symmetry Groups in Classical and Quantum Mechanics; Sov.J.Nucl.Phys. 4, 444 (1967).

[7] A.A.Makarov, Ya.A.Smorodinsky, Kh.Valiev and P.Winternitz. A Systematic Search for Nonrelativistic Systems with Dynamical Symmetries; Nuovo Cimento A 52, 1061 (1967).

[8] D.Bonatos, C.Daskaloyannis and K.Kokkotas. Deformed Oscillator Algebras for Two-Dimensional Quantum Superintegrable Systems; Phys. Rev. A 50, 3700 (1994).

[9] F.Calogero. Solution of a Three-Body Problem in One Dimension; J.Math.Phys. 10, 2191 (1969).

[10] L.G.Mardoyan, G.S.Pogosyan, A.N.Sissakian and V.M.Ter-Antonyan. Elliptic Basis for a Circular Oscillator. Nuovo Cimento, B 88, 43 (1985), Two-Dimensional Hydrogen Atom: I. Elliptic Bases; Theor.Math.Phys. 61, 1021 (1984); Hidden symmetry, Separation of Variables and Interbasis Expansions in the Two-Dimensional Hydrogen Atom. J.Phys., A 18, 455 (1985).

[11] Ya.A.Granovsky, A.S.Zhedanov and I.M.Lutzenko. Quadratic Algebra as a 'Hidden' Symmetry of the Hartmann Potential; J.Phys. A 24, 3887 (1991).

[12] C.Grosche, G.S.Pogosyan, A.N.Sissakian. Path Integral Discussion for Smorodinsky - Winternitz Potentials:I. Two - and Three Dimensional Euclidean Space. Fortschritte der Physik, 43, 453 (1995).

[13] P.Letourneau and L.Vinet. Superintegrable systems: Polynomial Algebras and Quasi-Exactly Solvable Hamiltonians. Ann. Phys. 243, 144168, (1995).

[14] E.G.Kalnins, W.Miller Jr. and G.S.Pogosyan. Superintegrability and associated polynomial solutions. Euclidean space and the sphere in two dimensions. J.Math.Phys. 37, 6439, (1996).

[15] M.F. Rañada. Superintegrable $n=2$ systems, quadratic constants of motion, and potentials of Drach. J.Math.Phys. 38, 4165, (1997). 
[16] W.Miller, Jr. Symmetry and Separation of Variables. Addison-Wesley Publishing Company, Providence, Rhode Island, 1977.

[17] E.G. Kalnins and W. Miller, Jr. Separation of variables on n-dimensional Riemannian manifolds 1. The n-sphere $S_{n}$ and Euclidean n-space $R_{n} J$. Math. Phys. 27, 1721, (1986).

[18] E.G. Kalnins. Separation of Variables for Riemannian Spaces of Constant Curvature, Pitman, Monographs and Surveys in Pure and Applied Mathematics 28, Longman, Essex, England, 1986.

[19] L. Landau and E. Lifshitz. The Classical Theory of Fields (Translated from Russian.) Addison-Wesley Publishing Company, Providence, Rhode Island, 1959.

[20] V.I. Arnold. Mathematical Methods of Classical Mechanics. (translated by K. Vogtmann and A. Weinstein) Graduate Texts in Mathematics, 60, Springer-Verlag, New York, 1978.

[21] W. Miller Jr. Mechanisms for variable separation in partial differential equations and their relationship to group theory. In Symmetries and Non-linear Phenomena pp. 188. World Scientific, 1988.

[22] N. Vilenkin. Special Functions and the Theory of Group Representations Amer. Math. Soc. Transl., Amer. Math. Soc., Providence, Rhode Island, 1968.

[23] W.Miller, Jr. Lie Theory and Special Functions. Academic Press, New York, 1968.

[24] E.G. Kalnins and W. Miller, Jr. $q$-algebra representations of the Euclidean, pseudo-Euclidean and oscillator algebras, and their tensor products. in Symmetries and Integrability of Difference Equations, Decio Levi editor, CRM Proc. Lect. Notes, American Mathematical Society, Providence, RI 1996.

[25] Lewis Carroll. Alice's Adventures in Wonderland. (1865) St. Martins Press, New York, 1991. 
[26] P. Winternitz, I. Lukac and Ya A. Smorodinskii. Quantum numbers of the little group of the Poincaré group. Soviet J. Nucl. Phys. 7, 139-145, (1968).

[27] V.N. Shapovalov. Stäckel spaces. Siberian Math. J., 20, 790-800, (1980).

[28] E.G. Kalnins and W. Miller, Jr. The theory of oprthogonal $R$-separation for Helmholtz equations. Advances in Mathematics 51, 91-106, (1984).

[29] A. Erdélyi et al. Higher Transcendental Functions, Vol. II. McGrawHill, New York, 1953.

[30] E.G. Kalnins, W. Miller and M.V. Tratnik. Families of orthogonal and biorthogonal polynomials on the $n$-sphere. SIAM J. Math. Anal., 22, 272-294, (1991).

[31] E.G. Kalnins and W. Miller. Hypergeometric expansions of Heun polynomials. SIAM J. Math. Anal., 22, 1450-1459, (1991).

[32] E.G. Kalnins, W. Miller and G.S. Pogosyan. Superintegrability in three dimensional Euclidean space. J.Math.Phys., 40, 708-725. (1999).

[33] Yu.N.Demkov: Symmetry Group of the Isotropic Oscillator. Sov.Phys.JETP 26 (1954) 757. Ibidem 36 (1959) 63-66.

[34] C.P.Boyer, E.G.Kalnins and P.Winternitz. Completely integrable relativistic Hamiltonian systems and separation of variables in Hermitian hyperbolic spaces. J.Math.Phys. 24, 2022 (1983).

[35] C.P. Boyer, E.G. Kalnins and P. Winternitz. Separation of variables for the Hamilton-Jacobi equation on complex projective spaces. SIAM J. Math. Anal. 16, 93-109, (1985).

[36] E.G. Kalnins, W. Miller and G.S. Pogosyan. Completeness of multiseparable superintegrability in $E_{2, C}$. IMA Preprint 1678 (submitted for publication).

[37] E.G. Kalnins, W. Miller and G.S. Pogosyan. Completeness of multiseparable superintegrability on the complex 2-sphere. IMA Preprint 1686 (submitted for publication). 